CERN-TH/2000-042

\title{
STRING COSMOLOGY: THE PRE-BIG BANG SCENARIO
}

\author{
G. Veneziano \\ Theoretical Physics Division, CERN \\ $\mathrm{CH}-1211$ Geneva 23
}

\begin{abstract}
A review is attempted of physical motivations, theoretical and phenomenological aspects, as well as outstanding problems, of the pre-big bang scenario in string cosmology.

Lectures given at the Summer School "The Primordial Universe"

Les Houches, July 1999
\end{abstract}

CERN-TH/2000-042

January 2000 


\section{Introduction}

These four lectures aim at providing a summary of -and some guidance through- the existing literature dealing with the so-called pre-big bang (PBB) scenario, a new cosmological model largely based on the new symmetries underlying superstring cosmology. The lectures will be pedagogical in nature and will not presuppose an advanced knowledge either of modern inflationary cosmology or of superstring/M-theory. Elements of both will be included in the lectures in order to make them reasonably self-contained. More exhaustive treatments of prebig bang cosmology are [1] (or will soon be [2]) available elsewhere, while a homepage on the PBB scenario is being kept updated on the Web [3].

The four lectures roughly correspond to the four forthcoming sections and deal, respectively, with:

- BASIC MOTIVATIONS AND IDEAS

- HOW COULD IT HAVE STARTED?

- PHENOMENOLOGICAL CONSEQUENCES

- HOW COULD IT HAVE STOPPED?

In particular, lecture II (Section 3) contains a discussion of the initial conditions, lecture III (Section 4) discusses the phenomenological virtues and shortcomings of the model, while lecture IV (Section 5) deals with the most important open theoretical issues.

\section{Basic Motivations and Ideas}

\subsection{Why string cosmology?}

The first question that comes to one's mind when thinking about cosmology and string theory is: Why bother? Indeed, even if string/M-theory is the correct theory of nature, only its effective (low-energy) quantum field theory description appears to be relevant to most of the history of our Universe, i.e. since a very short time after the big bang. This is certainly the case for the standard (hot-big-bang) cosmological model, but it is also true for the standard models of inflation, provided we confine our attention to what happened during the last 70 e-fold of inflation and later (i.e. to what happened after our present horizon reached the size of the inflationary Hubble radius). In both instances, one is only confronting situations in which curvatures are very small with respect to the fundamental scale of string theory.

On the other hand, both the hot-big bang model and its inflationary variant suffer from initial condition problems. In the former case, these are just the well-known homogeneity and flatness problems that motivated inflation. In the latter case, although the problems look less 
severe, it is still a matter of heated discussion whether or not one should naturally expect a quasi-homogeneous inflaton field highly displaced from the minimum of its potential to emerge from the Planck era. In either case, the question of how to get physically appealing initial conditions lies in the realm of Planck-scale quantum gravity.

At present, the only candidate for a consistent synthesis of general relativity (GR) and quantum mechanics (QM) is superstring theory (see [⿴囗 이 for a recent review, as well as [5] for a non-specialized introduction), or, if we prefer, the mysterious M-theory that reduces to various superstring theories in appropriate limits. It thus seems mandatory to ask whether the above questions on initial conditions do -or do not- find an answer within string theory. Although most string theorists would certainly agree with the above statements -this being after all one of the most selling ads for string theory- many of them would still object to tackling these problems now. The "excuse" is that our understanding of string theory, especially at large curvatures, is still largely incomplete. Furthermore, most of the recent progress in non-perturbative string theory has been achieved in the context of "vacua" (i.e. classical solutions to the field equations) that respect a large number of supersymmetries. By definition, a cosmological background (a fortiori one that evolves rapidly in time) breaks (albeit spontaneously) supersymmetry. This is why the Planckian regime of cosmology appears to be intractable for the time being.

There is however a pleasant surprise. About ten years of work on string cosmology have led naturally to considering a scenario -the so-called pre-big bang (PBB) scenario- in which the Universe enjoyed a long perturbative "life" before the big bang. Starting from an almost trivial state (asymptotic past triviality, see Section 3), the Universe would have evolved towards stronger and stronger curvature and coupling, thereby inflating, until it entered the non-perturbative phase that replaces the big bang singularity of more standard cosmological models.

The situation is very much reminiscent of QCD and strong interactions. Perturbative QCD has been very successful in predicting a huge number of observables for short-distancedominated hard processes. Successes in the non-perturbative, large-distance regime have been meagre, by comparison: we still lack a definitive proof of confinement, of spontaneous chiral symmetry breaking, of explicit $U(1)_{A}$ breaking, etc. Yet, we do believe that QCD is the correct description of hadronic physics down to scales of $10^{-15} \mathrm{~cm}$ or so. This is largely based on the belief that large- and short-distance physics "decouple", e.g. on the assumption that the soft hadronization process does not affect certain infrared-safe quantities computed at the quarkgluon level. Fortunately, we did not wait until the confinement problem was solved, to take QCD seriously!

A very similar attitude will be defended here in the case of string cosmology, with one amusing twist: large- and short-distance physics get somehow swapped as we go from QCD to gravity/cosmology. Figure 1 (from Ref. [6]) illustrates this point. The easy regime for gravity is at large distance/small curvatures; the tough one turns out to be the high-curvature regime that replaces here the big bang singularity. Yet, we shall argue that some consequences of string cosmology, those related to scales that were very large with respect to the string scale in the high-curvature regime, should not be affected, other than by a trivial kinematical red-shift, by 
the details of the pre- to post-big bang transition ... provided, of course, that such a transition does indeed take place (the counterpart to assuming that confinement does occur in QCD).

The above reasoning does not imply, of course, that one should not address the hard questions now. On the contrary, the easy part of the game will give precious information on what the relevant hard questions are (for cosmology) and on how to formulate them. I have already mentioned an example of what I mean: insisting too much on (extended) SUSY vacua appears to be an unacceptable limitation for the problems at hand. Another example is that of demanding stability of an acceptable string vacuum: we shall see (in Section 4) that inflationary string vacua lead to tachyonic, i.e. to growing rather than to oscillating, modes. Such modes appear to horrify most string theorists; however, they are just what inflationary cosmologists happily use all the time in order to generate large-scale structure (LSS), and what PBB cosmology uses to generate heat and entropy from an initially cold Universe (see Section 5).

A completely different criticism of string cosmology comes from the cosmology end: for someone accustomed to a data-driven "bottom-up" approach, string cosmology is too much "top-down". There is certainly a point here. I do not believe that a good model of cosmology is likely to emerge from theoretical considerations alone. Input from the data will be essential in the selection among various theoretical alternatives. We shall see explicit examples of what I mean in Section 5. Yet, it appears that a combination of top-down and bottom-up would be highly desirable. If past history can teach us something in this respect, the construction of the standard model of particle physics (and of QCD in particular) is a perfect example of a fruitful interplay of theoretically sound ideas and beautiful experimental results. Cosmology today resembles the particle physics of the sixties: interesting new data keep coming in at a high pace, while compelling theoretical pillars on which to base our understanding of those data are still missing.

As a final remark, let me turn things around and claim that cosmology could be the only hope that we have for testing string theory in the foreseeable future by using the cosmos itself as the largest conceivable accelerator. The cosmological red-shift since the big bang has kindly brought down Planck-scale physics to a macroscopic scale, thus opening for us a window on the very early Universe. As we shall see in Subsection 2.3, even in this respect, standard and PBB inflation are markedly different.

\subsection{Why/Which inflation?}

The reasons why the standard hot-big-bang model is unsatisfactory have been repeatedly discussed in the literature. For details, we refer to two excellent reviews [7]. Let me briefly summarize here the basic origin of those difficulties with the simplest Friedmann-RobertsonWalker (FRW) cosmology.

In the FRW framework the size of the (now observable) Universe was about $10^{-2} \mathrm{~cm}$ at the start of the classical era, say at $t \sim$ a few times $t_{P}$, where $t_{P} \sim 10^{-43} \mathrm{~s}$ is the so-called Planck

time. This is of course a very tiny Universe w.r.t. its present size $\left(\sim 10^{28} \mathrm{~cm}\right)$, yet it is huge 

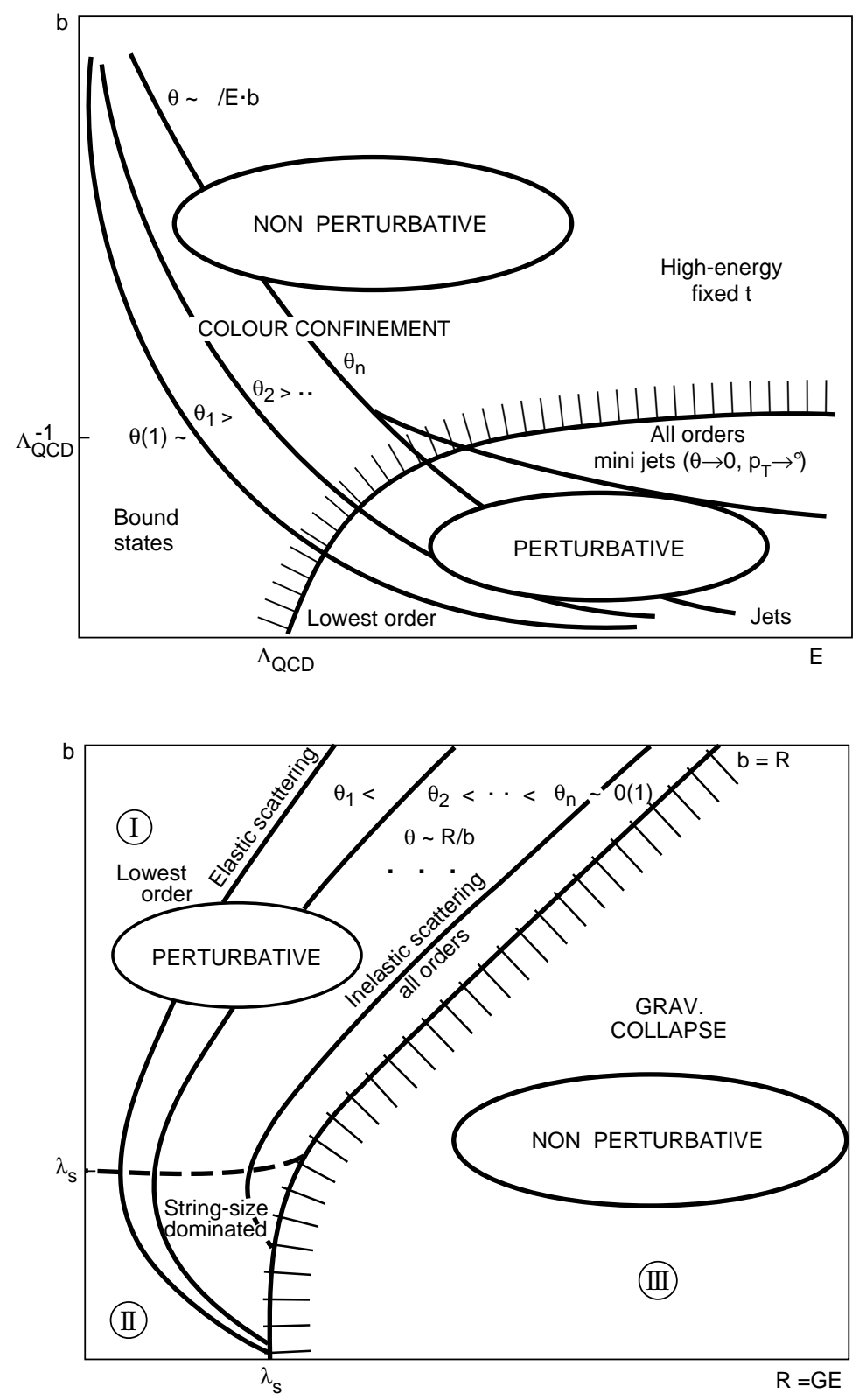

Figure 1 
w.r.t. the horizon (the distance travelled by light) at that time, i.e. to $l_{P}=c t_{P} \sim 10^{-33} \mathrm{~cm}$. In other words, a few Planck times after the big bang, our observable Universe was much too large! It consisted of $\left(10^{30}\right)^{3}=10^{90}$ Planckian-size, causally disconnected regions. There had not been, since the beginning, enough time for the Universe to become homogeneous (e.g. to thermalize) over its entire size. Also, soon after $t=t_{P}$, the Universe was characterized by a huge hierarchy between its Hubble radius on one side and its spatial-curvature radius on the other. The relative factor of (at least) $10^{30}$ appears as an incredible amount of fine-tuning on the initial state of the Universe, corresponding to a huge asymmetry between time and space derivatives. Was this asymmetry really there? And, if so, can it be explained in any, more natural way?

It should be stressed that, while the above unexplained ratio becomes larger and larger as we approach the Planck time (and would go to infinity at $t=0$ if we could trust the equations throughout), it represents the ratio of two classical length scales. It so happens that one of the two lengths becomes the (quantum) Planck scale at $t=t_{P}$, but the ratio is still huge at much later times when both scales have nothing to do with (and are much larger than) $t_{P}$. This comment will be very relevant to the discussion of fine-tuning issues given in Subsection 3.6.

It is well known that a generic way to wash out inhomogeneities and spatial curvature consists in introducing, in the history of the Universe, a long period of accelerated expansion, called inflation [7]. This still leaves two alternatives: either the Universe was generic at the big bang and became flat and smooth because of a long post-bangian inflationary phase; or it was already flat and smooth at the big bang as a result of a long pre-bangian inflationary phase.

Assuming, dogmatically, that the Universe (and time itself) started at the big bang, leaves only the first alternative. However, that solution has its own problems, in particular those of fine-tuned initial conditions and inflaton potentials. Besides, it is quite difficult 8 to base standard inflation in the only known candidate theory of quantum gravity, superstring theory. Rather, as we shall argue in a moment, superstring theory gives strong hints in favour of the second (pre-big bang) possibility through two of its very basic properties, the first in relation to its short-distance behaviour, the second from its modifications of GR even at large distance.

\subsection{Superstring-inspired cosmology}

As just mentioned, two classes of properties of string theory are relevant for cosmology. Let us discuss them in turn.

\section{A) Short-distance properties}

Since the classical (Nambu-Goto) action of a string is proportional to the area $A$ of the surface it sweeps, its quantization must introduce a quantum of length $\lambda_{s}$ through:

$$
S / \hbar=A / \lambda_{s}^{2} .
$$

This fundamental length, replacing Planck's constant in quantum string theory [9], plays the 
role of a minimal observable length, of an ultraviolet cut-off. Thus, in string theory, physical quantities are expected to be bound by appropriate powers of $\lambda_{s}$, e.g.

$$
\begin{array}{r}
H^{2} \sim R \sim G \rho<\lambda_{s}^{-2} \\
k_{B} T / \hbar<c \lambda_{s}^{-1} \\
R_{\text {comp }}>\lambda_{s} .
\end{array}
$$

In other words, in quantum string theory, relativistic quantum mechanics should solve the singularity problems in much the same way as non-relativistic quantum mechanics solved the singularity problem of the hydrogen atom by keeping the electron and the proton a finite distance apart. By the same token, string theory gives us a rationale for asking daring questions such as: What was there before the big bang? Certainly, in no other present theory can such a question be meaningfully asked.

\section{B) Large-distance properties}

Even at large distance (low-energy, small curvatures), superstring theory does not automatically give Einstein's GR. Rather, it leads to a scalar-tensor theory of the JBD variety. The new scalar particle/field $\phi$, the so-called dilaton, is unavoidable in string theory, and gets reinterpreted as the radius of a new dimension of space in so-called M-theory [10]. By supersymmetry, the dilaton is massless to all orders in perturbation theory, i.e. as long as supersymmetry remains unbroken. This raises the question: Is the dilaton a problem or an opportunity? My answer is that it could be both; and while we can try to avoid its potential dangers, we may try to use some of its properties to our advantage ... Let me discuss how.

In string theory, $\phi$ controls the strength of all forces [11], gravitational and gauge alike. One finds, typically:

$$
l_{P}^{2} / \lambda_{s}^{2} \sim \alpha_{\text {gauge }} \sim e^{\phi},
$$

showing the basic unification of all forces in string theory and the fact that, in our conventions, the weak-coupling region coincides with $\phi \ll-1$. In order not to contradict precision tests of the equivalence principle, and of the constancy of the gauge and gravitational couplings in the "recent" past, we require [12] the dilaton to have a mass (see, however, [13] for an amusing alternative) and to be frozen at the bottom of its own potential today. This does not exclude, however, the possibility of the dilaton having evolved cosmologically (after all, the metric did!) within the weak coupling region where it was practically massless. The amazing (yet simple) observation [14] is that, by so doing, the dilaton may have inflated the Universe!

A simplified argument, which, although not completely accurate, captures the essential physical point, consists in writing the Friedmann equation (for a spatially flat Universe):

$$
3 H^{2}=8 \pi G \rho
$$

and in noticing that a growing dilaton (meaning through (3) a growing $G$ ) can drive the growth of $H$ even if the energy density of standard matter decreases in an expanding Universe. This new 


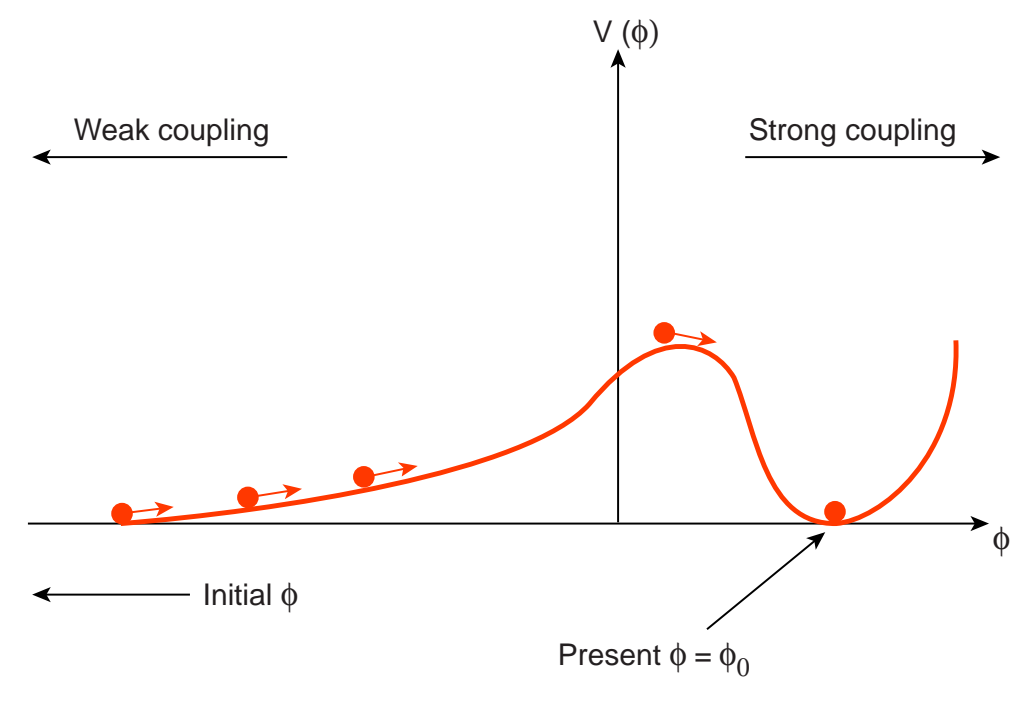

Figure 2

kind of inflation (characterized by growing $H$ and $\phi$ ) has been termed dilaton-driven inflation (DDI). The basic idea of pre-big bang cosmology [14, 15, 16, 17] is thus illustrated in Fig. 2: the dilaton started at very large negative values (where it was practically massless), ran over a potential hill, and finally reached, sometime in our recent past, its final destination at the bottom of its potential $\left(\phi=\phi_{0}\right)$. Incidentally, as shown in Fig. 2, the dilaton of string theory can easily roll-up — rather than down - potential hills, as a consequence of its non-standard coupling to gravity.

DDI is not just possible. It exists as a class of (lowest-order) cosmological solutions thanks to the duality symmetries of string cosmology [14], [18], [19]. Under a prototype example of these symmetries, the so-called scale-factor duality (SFD) [14, [18, a FRW cosmology evolving (at lowest order in derivatives) from a singularity in the past is mapped into a DDI cosmology going towards a singularity in the future. Of course, the lowest order approximation breaks down before either singularity is reached. A (stringy) moment away from their respective singularities, these two branches can easily be joined smoothly to give a single non-singular cosmology, at least mathematically. Leaving aside this issue for the moment (see Section 5 for more discussion), let us go back to DDI. Since such a phase is characterized by growing coupling and curvature, it must itself have originated from a regime in which both quantities were very small. We take this as the main lesson/hint to be learned from low-energy string theory by raising it to the level of a new cosmological principle, that of "Asymptotic Past Triviality", to be discussed in the next Lecture.

\subsection{Explicit solutions}

Many explicit exact PBB-type solutions to the low-energy effective action equations have been constructed and discussed in the literature. For an excellent review, see [1]. Exact solutions can 
only be obtained in the presence of symmetries (isometries) and, although they are heuristically very important, they are too special from the point of view of an inflationary cosmology, which, as such, should not accept fine-tuned initial conditions. This is why we shall not go into an exhaustive discussion of explicit solutions here. Instead, in Section 3, we will adress the general problem of the evolution of asymptotically trivial initial data.

Here we shall limit our attention to the simplest Bianchi I-type solutions and to their quasihomogeneous generalizations, after recalling that many more solutions can be obtained from the former by using the non-compact $O(d, d)$ symmetry of the low-energy string-cosmology equations [19] when the Kalb-Ramond (KR) field $B_{\mu \nu}$ is turned on, or by S-duality transformations (see e.g. [1]) generating a homogeneous axion field (related to $B_{\mu \nu}$ by yet another duality transformation).

The generic homogeneous Bianchi I solution with $B_{\mu \nu}=0$ reads, for $t<0$,

$$
\begin{aligned}
d s^{2} & =-d t^{2}+\sum_{i}(-t)^{2 \alpha_{i}} d x^{i} d x^{i} \\
\phi & =-\left(1-\sum_{i} \alpha_{i}\right) \log (-t) \\
1 & =\sum_{i} \alpha_{i}^{2}
\end{aligned}
$$

i.e. represents a generalization of the well-known Kasner solutions (see e.g. [20]) in which one of the two Kasner constraints (the one linear in the $\alpha_{i}$ ) is replaced by the equation giving the time dependence of $\phi$ ( $\phi$ is absent, or constant, for Kasner, hence the second constraint).

Note that, unlike Kasner's, (可) allows for isotropic solutions $\left(\alpha_{i}= \pm 1 / \sqrt{d}\right.$ for all $\left.i\right)$. Also, the quadratic Kasner constraint automatically has $2^{d}$ SFD-related branches, obtained by changing the sign of any subset of the $\alpha^{\prime} s$. Also note that the so-called shifted dilaton defined by:

$$
\bar{\phi}=\phi-\frac{1}{2} \log \left(\operatorname{det} g_{i j}\right)
$$

which is invariant under the full $O(d, d)$ group, is always given by:

$$
\bar{\phi}=-\log (-t) \text {. }
$$

A quasi-homogeneous generalization of (5) was first discussed in [21] (see also [22]) and reads:

$$
\begin{aligned}
d s^{2} & =-d t^{2}+\sum_{a} e_{i}^{a}(x) e_{j}^{a}(x)(-t)^{2 \alpha_{a}(x)} d x^{i} d x^{j} \\
\phi & =-\left(1-\sum_{i} \alpha_{i}(x)\right) \log (-t) \\
1 & =\sum_{i} \alpha_{i}^{2}(x) \quad, \quad t<0
\end{aligned}
$$

where $x$ stands for the space coordinates. Equation (8) can be shown to be a generic asymptotic solution of the full PDEs near the $t=0$ singularity where spatial gradients become less and 


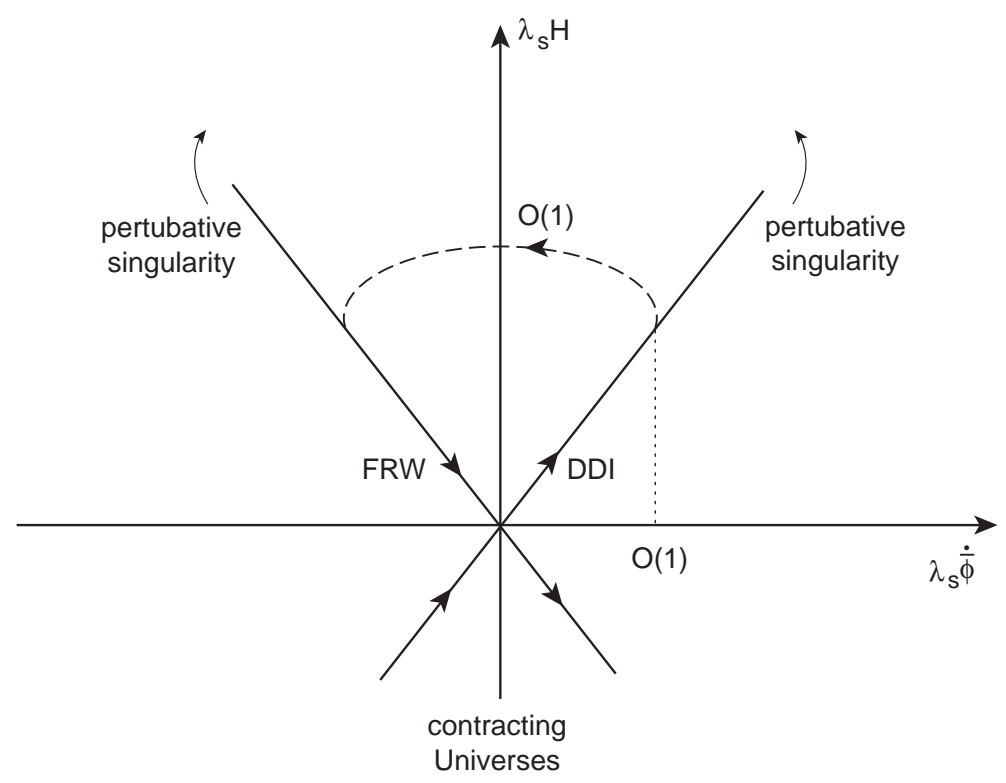

Figure 3

less important w.r.t. time derivatives, justifying the validity of the so-called gradient expansion [23]. Note that Eq. (77) is not modified in the quasi-homogeneous solutions. Besides allowing isotropic cosmologies in the homogeneous case, the presence of the dilaton also removes the necessity of a chaotic (BKL-type [24]) behaviour near the singularity [25].

\subsection{Phase diagrams and Penrose-style overview}

It is useful to visualize the PBB scenario with the help of some diagrams. Since the actual phase space of the model is multidimensional, each of these diagrams necessarily represents just a cross section of the complete picture.

A very commonly used diagram (Fig. 3 ) is the flow-diagram in the $\dot{\bar{\phi}}, H$ plane (time being just a parameter along the flow lines). Since, at lowest order, $\ddot{\bar{\phi}} \geq 0$, the flow is always from left to right near the origin. The four straight lines represent the four (isotropic for simplicity) solutions connected by SFD and time-reversal. The product of the two transformations represents the physically interesting case, since it maps ordinary decelerating FRW cosmology (top left) to dilaton-driven inflation (top right). Clearly, our scenario needs a high-curvature phase during which the left-to-right flow is inverted (as shown by the dotted line joining the two perturbative branches). This can only happen as the result of higher-order corrections (see Section 5).

A second useful diagram (Fig. 4) is the $e^{\phi}, H$ plot, i.e. the curvature (energy) coupling plane. The fully perturbative domain (where evolution starts according to the APT postulate) lies, in a log-log plot, to the far left-bottom corner. Sticking again, for simplicity, to the isotropic 


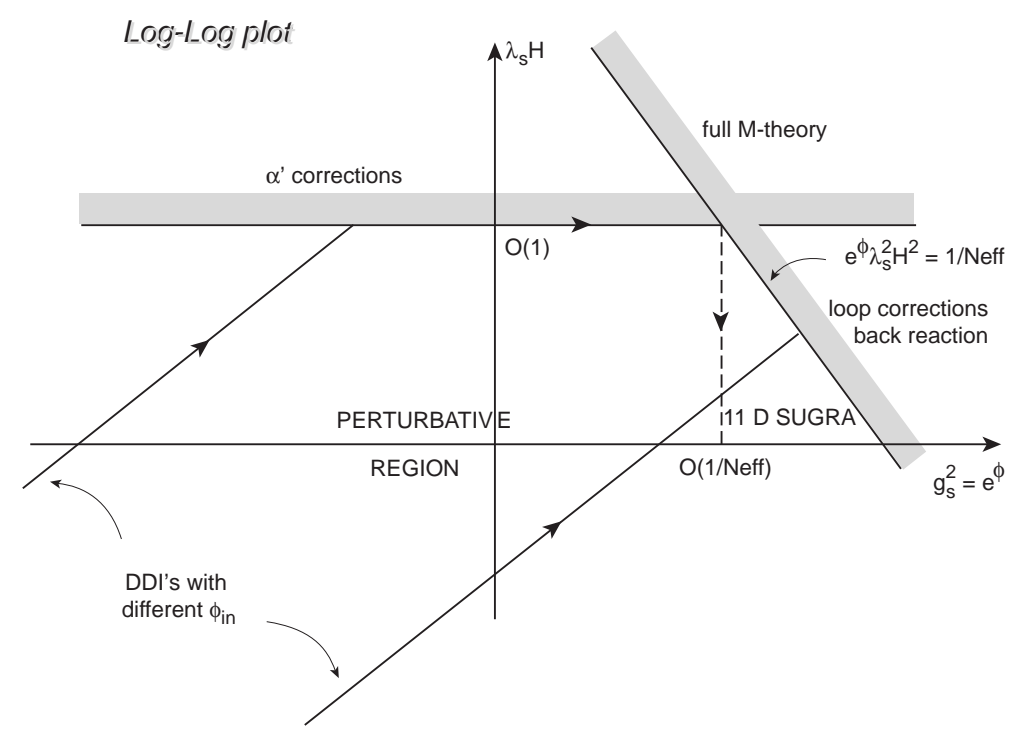

Figure 4

case, DDI evolution is represented by parallel lines distinguished by different initial values of the dilaton (i.e. of the coupling). It is clear that all these solutions run, eventually, into strong curvature or strong coupling (shown as thick solid lines), which one is hit first being determined by the above-mentioned initial coupling. A discussion of what might happen afterwards is given in Section 5.

As a third possibility, let us use a Carter-Penrose style plot [26] (Fig. 5) to represent, on a finite piece of paper, the entire evolution of the Universe. Unlike in ordinary cosmology, where the CP diagram is truncated by the (space-like) hypersurface of the big-bang singularity, here the whole CP diagram, going from past to future time-like and null infinities, is physically meaningful because of our assumption that finite-string-size effects remove the big bang singularity. This diagram will be discussed and used in the following sections.

Finally, let us represent the basic difference between the standard inflation scenario and that of PBB cosmology by plotting, for each cosmological model, the Hubble horizon $\left(H^{-1}\right)$ and the physical scale that coincides with it today, as functions of cosmic time. This gives rise to two "wine glasses" (Fig. 6), which are very similar in their upper parts (corresponding to recent epochs) but differ markedly at very early times. The most salient difference appears in the early behaviour of the Hubble horizon, an increasing function of time in the standard inflation, a decreasing one in the PBB case. The figure allows me to stress one phenomenological advantage of PBB inflation: Planck- (or string)-scale physics, being no longer washed out by a long, subsequent inflationary phase, becomes accessible to present (or near-future) experiments at the millimetre $(100 \mathrm{GHz}$ ) scale. At the same time, larger-scale experiments (such as those on small-angle CMB anisotropies) will test (sub-Planckian-energy) physics during the pre-bangian phase. By contrast, as we have already mentioned in the Introduction, in standard inflation large-scale data probe the Universe as it was seventy e-folds or so before the end of inflation, 


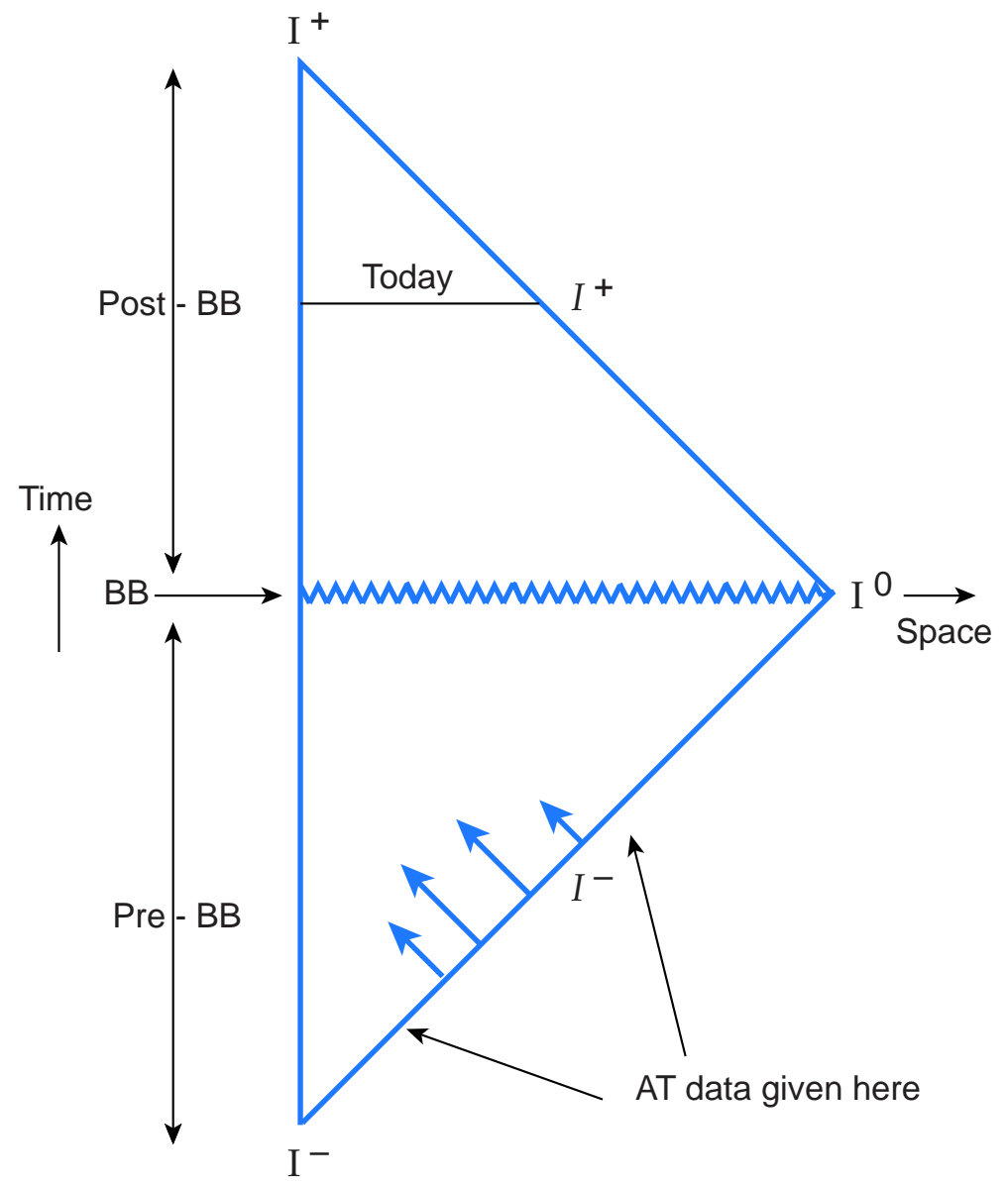

Figure 5 

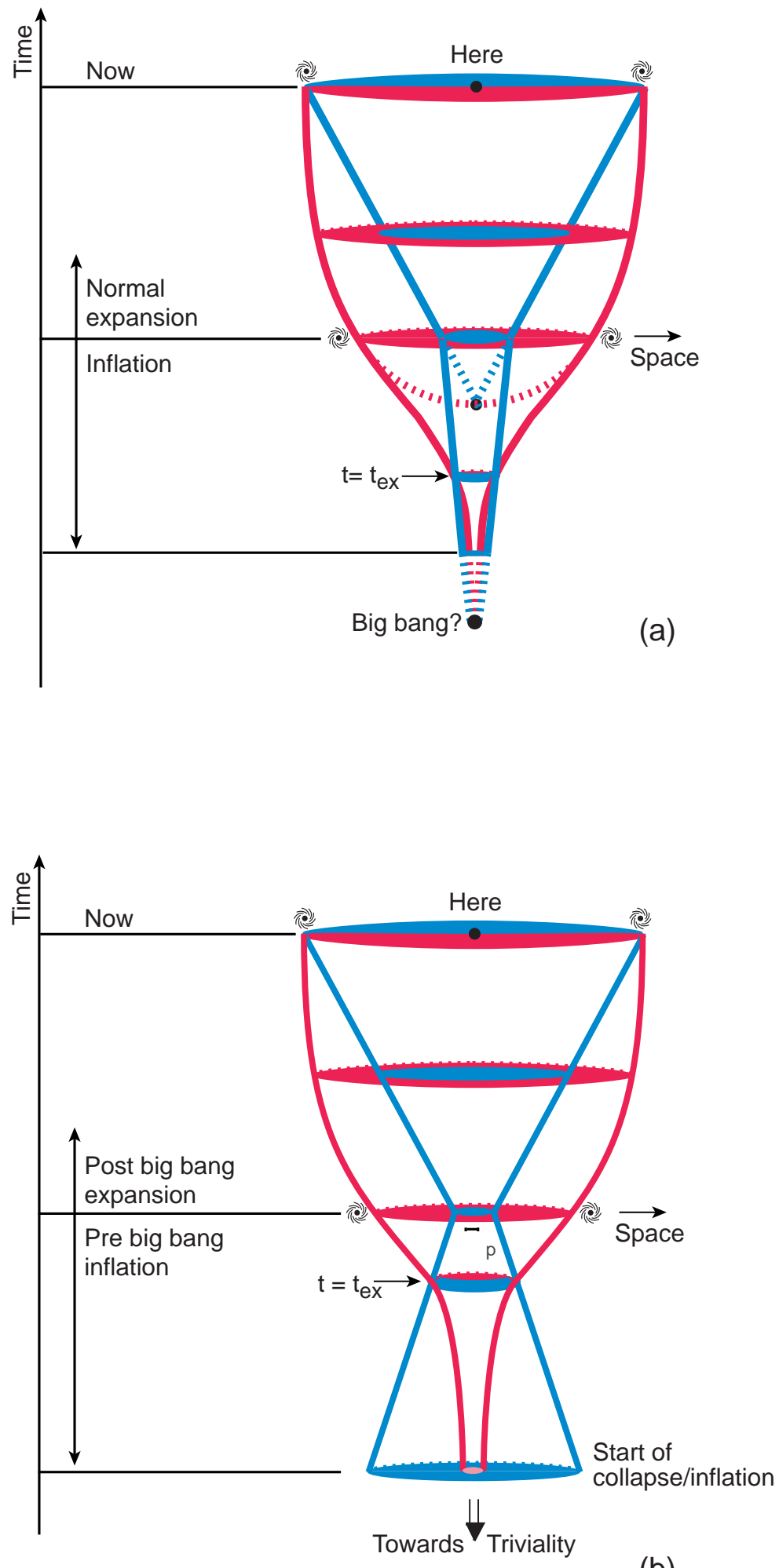

Figure 6

(a) Standard inflation's wineglass; (b) Pre-Big Bang's wineglass 
while shorter scales tells us about more recent epochs. Since we know that, seventy e-folds before the end of inflation, $H_{\text {infl }}$ was less than $10^{-5} M_{P}$ (or else excessive large scale anisotropies are created, see Section 4), and that such a scale slowly decreases during (slow-roll) inflation, it is clear that, according to standard inflation, physics at energies larger than $10^{-5} M_{P}$ remains unaccessible.

\section{How could it have started?}

\subsection{Generic asymptotically-trivial past}

We have already mentioned that, in standard non-inflationary cosmology, initial conditions have to be fine-tuned to incredible accuracy in the far past (i.e. at $t \sim t_{P} \sim 10^{-43} \mathrm{~s}$ ). What does this fine-tuning problem look like if we accept hints from scale-factor duality and assume asymptotically trivial, yet generic, initial conditions?

The concept of asymptotic past triviality (APT) is quite similar to that of "asymptotic flatness", familiar from general relativity [27]. The main differences consist in making only assumptions concerning the asymptotic past (rather than future or space-like infinity) and in the additional presence of the dilaton. It seems physically (and philosophically) satisfactory to identify the beginning with simplicity (see e.g. the entropy-related arguments given in Subsection 5.7). What could be simpler than a trivial, empty and flat Universe? Nothing, of course! The problem is that such a Universe, besides being uninteresting, is also non-generic. By contrast, asymptotically flat/trivial Universes are initially simple, yet generic, in a precise mathematical sense that we shall now discuss.

From the point of view of space-time (taken here, for simplicity, to be $(3+1)$-dimensional) the generic solution depends upon four arbitray functions of three coordinates [28] related to the metric, plus two more each for the dilaton and the KR field $B_{\mu \nu}$. Amusingly, there is an exact correspondence between this "target-space" counting and a "world-sheet" counting. In the latter, those eight arbitrary functions correspond to eight arbitrary functions of three-momentum entering the most general physical (i.e. on shell) vertex operator describing gravitons, dilatons, and the KR field (which, in four dimensions, is equivalent to a pseudoscalar, the KR axion). We will see in Subsection 3.4 how these arbitrary functions appear in the asymptotic expansion of our fields.

Can a very rich and complicated Universe, like our own, emerge from such extremely simple initial conditions? This would look much like a miracle. However, as I shall argue below, this is precisely what should be expected, owing to well-known classical and quantum gravitational instabilities. 


\subsection{The asymptotic past's effective action and different (conformal) frames}

The APT postulate implies that the early-time evolution of the Universe can be described in terms of the low-energy tree-level action of string theory. Taking a generic closed superstring theory, this reads:

$$
\Gamma_{e f f}=\lambda_{s}^{1-d} \int d^{d+1} x \sqrt{|g|} e^{-\phi}\left(R+g^{\mu \nu} \partial_{\mu} \phi \partial_{\nu} \phi-\frac{1}{12}(d B)^{2}-2 \Lambda\right),
$$

where $d B$ is the (three-form) field strength associated with $B_{\mu \nu}$.

A further simplification comes from assuming to be dealing with so-called critical superstring theory, the case in which the tree-level (and actually the all-order perturbative) cosmological constant $\Lambda$ vanishes. This requires a total of $D=10$ space-time dimensions. If $D \neq 10$ there will be an effective cosmological constant $O\left(\lambda_{s}^{-2}\right)$ preventing any low-curvature solution of the field equations to exist. A similar conclusion is reached if we consider critical, but non-supersymmetric, string theories (see Subsection 3.3).

Equation (9) receives corrections when curvatures become $O\left(\lambda_{s}^{-2}\right)$ or when the coupling $e^{\phi}$ becomes $O(1)$. If such corrections are both negligible, it sometimes becomes useful to perform a change of variable by going to the so-called Einstein frame (not to be confused with different frames in GR). This is done by defining:

$$
g_{\mu \nu}=g_{\mu \nu}^{(E)} e^{\frac{2}{d-1}\left(\phi-\phi_{0}\right)} .
$$

It is relatively easy to rewrite the action (9) using the Einstein metric. The result is simply:

$$
\Gamma_{e f f}^{E}=l_{P}^{1-d} \int d^{d+1} x \sqrt{\mid g^{(E) \mid}}\left(R-\frac{1}{d-1} \partial_{\mu} \phi \partial^{\mu} \phi-\frac{1}{12} e^{-\frac{4}{d-1} \phi}(d B)^{2}\right),
$$

where $l_{P}^{d-1}=e^{\phi_{0}} \lambda_{s}^{d-1}$ is the present value of the Planck length.

Although the use of the Einstein frame could simplify some calculations, and we shall see examples of this below, it should be kept in mind that the form of the corrections is no longer so simple. For instance, higher-derivative corrections become important when the Einstein-frame curvature is $O\left(l_{P}^{-2} e^{-\frac{2}{d-1} \phi}=\lambda_{s}^{-2}\right)$, i.e. reaches a dilaton-dependent critical value. Similarly, having a constant Newton "constant" in this frame is a mere illusion because (even tree-level) string masses do now depend upon $\phi$. For these reasons, although physical results are frameindependent, we shall always describe them with reference to the original string-frame metric in which the stringh length $\lambda_{s}$ is constant.

Let us finally remark that the two frames have been made to coincide today, with the dilaton fixed at its present value $\phi_{0}$. Similarly, the assumption of APT would also allow the identification of the two frames in the far past, since the dilaton approaches a constant as $t \rightarrow-\infty$. However, the two Einstein frames that coincide with the string frame at $t= \pm \infty$ differ from each other by an enormous conformal factor, i.e. by a huge blowing-up of all physical scales. 


\subsection{Classical asymptotic symmetries: the importance of SUSY}

The classical equations that follow from varying (9) or (11), besides being generally covariant, are also invariant under a two-parameter group of (global) transformations acting as follows:

$$
\begin{aligned}
\phi & \rightarrow \phi+c, \\
g_{\mu \nu} & \rightarrow \lambda^{2} g_{\mu \nu} .
\end{aligned}
$$

Indeed (9), (11) are simply rescaled by a constant factor under this group. These two symmetries depend crucially on the validity of the tree-level low-energy approximation and on the absence of a cosmological constant. Loop corrections clearly spoil invariance under dilaton shifts, while lower derivatives (a cosmological constant) or higher derivatives $\left(\alpha^{\prime}\right)$ corrections spoil invariance under a rescaling of the metric. Note that, using general covariance, the latter symmetry is

equivalent to an overall rescaling of all the coordinates. The relevance of the two classical symmetries on the issue of fine-tuning will become obvious in the next two subsections.

The importance of dealing with critical superstring theory now becomes evident: if one would consider non-supersymmetric string theories, a cosmological constant would almost certainly be generated at some finite order of the loop expansion: this would change completely the large-distance properties and spoil the symmetries of the field equations.

\subsection{Dilaton-driven inflation as gravitational collapse}

For simplicity, we will only illustrate here the simplest case of gravi-dilaton system already compactified to four space-time dimensions. Through the field redefinition (10), our problem is reduced to the study of a massless scalar field minimally coupled to gravity. It is well known that such a form of matter cannot give inflation (since it has positive pressure). Instead, it can easily lead to gravitational collapse (GC). Thus, in the Einstein frame, the problem becomes that of finding out under which conditions gravitational collapse occurs if asymptotically-trivial initial data are assigned. Gravitational collapse usually means that the (Einstein) metric (hence the volume of 3-space) shrinks to zero at a space-like singularity. However, typically, the dilaton blows up at that same singularity. Given the relation (10) between the Einstein and the (physical) string metric, we can easily imagine that the latter blows up near the singularity, as implied by DDI.

How generically does GC happen? Let us recall the singularity theorems of Hawking and Penrose [29], which state that, under some general assumptions, singularities are inescapable in GR. Looking at the validity of those assumptions in the case at hand, one finds that all but one are automatically satisfied. The only condition to be imposed is the existence of a closed trapped surface (CTS) (a closed surface from which future light cones lie entirely in the region inside the surface). Rigorous results [30] show that this condition cannot be waived: sufficiently weak initial data do not lead to closed trapped surfaces, to collapse, or to singularities. Sufficiently 
strong initial data do. But where is the border-line? This is not known in general, but precise criteria do exist for particularly symmetric space-times, e.g. for those endowed with spherical symmetry (see Subsection 3.6).

However, no matter what the general collapse/singularity criterion will eventually turn out to be, we do know, from the classical symmetries described in the previous subsection, that such a criterion cannot depend

- on an over-all additive constant in $\phi$, or

- on an over-all multiplicative factor in $g_{\mu \nu}$.

A characterization of APT initial data can be made [31] following the pioneering work [27] of Bondi, Sachs, Penrose, and others. Since our initial quanta are assumed to consist of massless gravitons and dilatons, their past infinity is null: it is the famous $\mathcal{I}^{-}$of the Penrose diagram (Fig. 5). APT means that dilaton and metric can be expanded near $\mathcal{I}^{-}$in inverse powers of $r \rightarrow \infty$, while advanced time $v$ and two angular variables, $\theta$ and $\varphi$, are kept fixed. We shall thus write:

$$
\begin{gathered}
\phi\left(x^{\lambda}\right)=\phi_{0}+\frac{f(v, \theta, \varphi)}{r}+o\left(\frac{1}{r}\right), \\
g_{\mu \nu}\left(x^{\lambda}\right)=\eta_{\mu \nu}+\frac{f_{\mu \nu}(v, \theta, \varphi)}{r}+o\left(\frac{1}{r}\right) .
\end{gathered}
$$

The null wave data on $\mathcal{I}^{-}$are: the asymptotic dilatonic wave form $f(v, \theta, \varphi)$, and two polarization components, $f_{+}(v, \theta, \varphi)$ and $f_{\times}(v, \theta, \varphi)$, of the asymptotic gravitational wave form $f_{\mu \nu}(v, \theta, \varphi)$, whose other components can be gauged away. The three functions $f, f_{+}, f_{\times}$of $v, \theta, \varphi$ are equivalent to six functions of $r, \theta, \varphi$ with $r \geq 0$, because the advanced time $v$ ranges over the full line $(-\infty,+\infty)$. This is how the six arbitrary functions of the generic solution to the gravi-dilaton system are recovered.

Of particular interest here are the so-called News functions, simply given by

$$
N(v, \theta, \varphi) \equiv \partial_{v} f(v, \theta, \varphi), N_{+} \equiv \partial_{v} f_{+}, N_{\times} \equiv \partial_{v} f_{\times}
$$

and the "Bondi mass" given by:

$$
\begin{aligned}
M_{-}(v) & =\frac{1}{4 \pi} \int d^{2} \Omega M_{-}(v, \theta, \varphi), \\
g_{v v} & =-\left(1-\frac{2 M_{-}(v, \theta, \varphi)}{r}\right)+o\left(\frac{1}{r}\right) .
\end{aligned}
$$

The Bondi mass and the News are connected by the energy-momentum conservation equation, which tells us that the advanced-time derivative of $M_{-}(v)$ is positive-semidefinite and related to incoming energy fluxes controlled by the News:

$$
d M_{-}(v) / d v=\frac{1}{4} \int d^{2} \Omega\left(N^{2}+N_{+}^{2}+N_{\times}^{2}\right) .
$$


The physical meaning of $M_{-}(v)$ is that it represents the energy brought into the system (by massless sources) by advanced time $v$. In the same spirit one can define the Bondi mass $M_{+}(u)$ at future null infinity $\mathcal{I}^{+}$. It represents the energy still present in the system at retarded time $u$. If only massless sources are present, the so-called ADM mass is given by

$$
M_{-}(+\infty)=M_{+}(-\infty)=M_{A D M}
$$

while $M_{-}(-\infty)=0$, and $M_{+}(+\infty)=M_{C}$ represents the mass that has not been radiated away even after waiting an infinite time, i.e. the mass that underwent gravitational collapse [32]. Collapse (resp. no-collapse) criteria thus aim at establishing under which initial conditions one expects to find $M_{C}>0$ (resp. $\left.M_{C}=0\right)$.

Since, as we shall see in the particular case of spherical symmetry, collapse criteria i) do not involve any particularly large number, and ii) do not contain any intrinsic scale but just dimesionless ratios of various classical scales, we expect i) gravitational collapse to be quite a generic phenomenon and ii) that nothing, at the level of our approximations, will be able to fix either the size of the horizon or the value of $\phi$ at the onset of collapse. Generically, and quite randomly and chaotically, some regions of space will undergo gravitational collapse, will form horizons and singularities therein. When this is translated into the string frame, the region of space-time within the horizon undergoes a period of DDI in which both the initial value of the Hubble parameter and that of $\phi$ are left arbitrary. In the next subsection we shall see that such arbitrariness provides an answer to the fine-tuning allegations that have been recently

moved [33] to the PBB scenario. This section will be concluded with a discussion of how more precisely the case of spherical symmetry can be dealt with.

\subsection{Fine-tuning issues}

The two arbitrary parameters discussed in the previous subsection are very important, since they determine the range of validity of our description. In fact, since both curvature and coupling increase during DDI, the low-energy and/or tree-level description is bound to break down at some point. The smaller the initial Hubble parameter (i.e. the larger the initial horizon size) and the smaller the initial coupling, the longer we can follow DDI through the effective action equations and the larger the number of reliable e-folds we shall gain.

This does answer, in my opinion, the objections raised recently [33] to the PBB scenario according to which it is fine-tuned. The situation here actually resembles that of chaotic inflation [34]. Given some generic (though APT) initial data, we should ask which is the distribution of sizes of the collapsing regions and of couplings therein. Then, only the "tails" of these distributions, i.e. those corresponding to sufficiently large, and sufficiently weakly coupled, regions will produce Universes like ours, the rest will not. The question of how likely a "good" big bang is to take place is not very well posed and can be greatly affected by anthropic considerations [31].

In conclusion, we may summarize recent progress on the problem of initial conditions by saying that [31: 


\section{Dilaton-Driven Inflation in String Cosmology \\ is as generic as \\ Gravitational Collapse in General Relativity.}

Furthermore, asking for a sufficiently long period of DDI amounts to setting upper limits on two arbitrary moduli of the classical solutions.

Figure 7 (from Ref. [31]) gives a $(2+1$ )-dimensional sketch of a possible PBB Universe: an original "sea" of dilatonic and gravity waves leads to collapsing regions of different initial size, possibly to a scale-invariant distribution of them. Each one of these collapses is reinterpreted, in the string frame, as the process by which a baby Universe is born after a period of PBB inflationary "pregnancy", the size of each baby Universe being determined by the duration of the corresponding pregnancy, i.e. by the initial size of (and coupling in) the corresponding collapsing region. Regions initially larger than $10^{-13} \mathrm{~cm}$ can generate Universes like ours, smaller ones cannot.

A basic difference between the large numbers needed in (non- inflationary) FRW cosmology and the large numbers needed in PBB cosmology should be stressed. In the former, the ratio of two classical scales, e.g. of total curvature to its spatial component, which is expected to be $O(1)$, has to be taken as large as $10^{60}$. In the latter, the above ratio is initially $O(1)$ in the collapsing/inflating region, and ends up being very large in that same region, thanks to DDI. However, the (common) order of magnitude of these two classical quantities is a free parameter, and it is taken to be much larger than the classically irrelevant quantum scale. Indeed, the smallness of quantum corrections (which would introduce a scale in the problem) was explicitly checked in 35.

We can visualize analogies and differences between standard and pre-big bang inflation by looking again at Figs. $6 \mathrm{a}$ and $6 \mathrm{~b}$. The common feature in the two pictures is that the fixed comoving scale corresponding to the present horizon was "inside the horizon" for some time during inflation, possibly very deeply inside at its onset. The difference between the two scenarios is just in the behaviour of the Hubble radius during inflation: increasing in standard inflation (a), decreasing in string cosmology (b). Thus, while standard inflation is still facing the initial-singularity question and needs a non-adiabatic phenomenon to reheat the Universe (a kind of small bang), PBB cosmology faces the singularity problem later, combining it with the exit and heating problems (see Section 5).

\subsection{The spherically symmetric case}

In the spherically symmetric case many authors have studied the problem of gravitational collapse of a minimally coupled scalar field both numerically and analytically. In the former case I will only mention the well-known results of Choptuick [36], pointing at mysterious universalities near critical collapse (i.e. at the border-line situation in which the collapse criteria are just barely met). In this case, a very small black hole forms. This is not the case we are really interested in for the reasons we just explained. We shall thus turn, instead, to what happens 


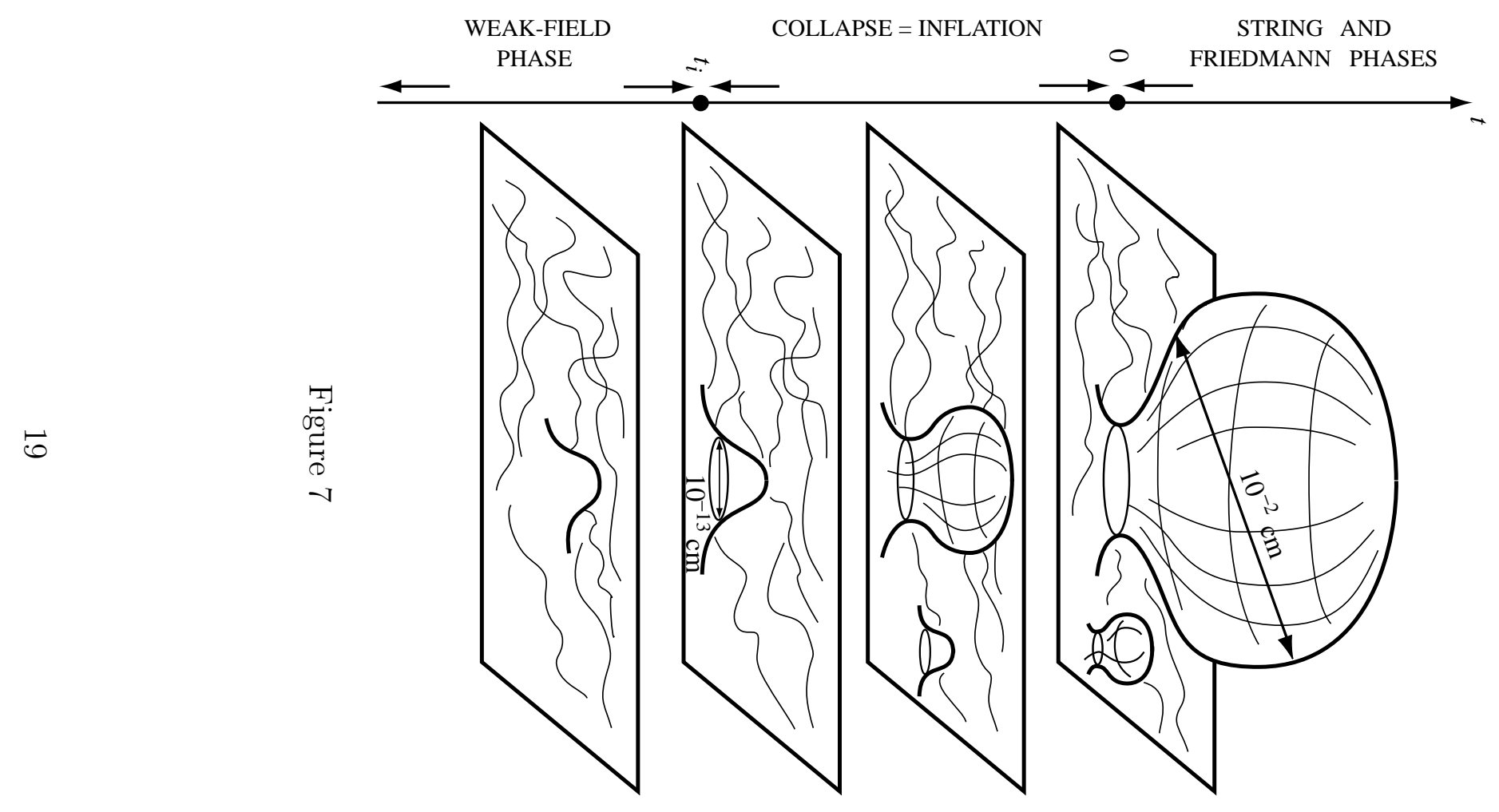


when the collapse criteria are largely fulfilled. For this we make use of the rather powerful results due to Christodoulou over a decade of beautiful work [30, [32], 37], 38].

There are no gravitational waves in the spherically symmetric case so that null wave data consist of just an angle-independent asymptotic dilatonic wave form $f(v)$, with the associated scalar News $N(v)=f^{\prime}(v)$.

A convenient system of coordinates is the double null system, $(u, v)$, such that

$$
\begin{gathered}
\phi=\phi(u, v), \\
d s^{2}=-\Omega^{2}(u, v) d u d v+r^{2}(u, v) d \omega^{2},
\end{gathered}
$$

where $d \omega^{2}=d \theta^{2}+\sin ^{2} \theta d \varphi^{2}$. The field equations are conveniently re-expressed in terms of the three functions $\phi(u, v), r(u, v)$ and $m(u, v)$, where the local mass function $m(u, v)$ is defined by:

$$
1-\frac{2 m}{r} \equiv g^{\mu \nu}\left(\partial_{\mu} r\right)\left(\partial_{\nu} r\right)=-\frac{4}{\Omega^{2}} \frac{\partial r}{\partial u} \frac{\partial r}{\partial v}
$$

One gets the following set of evolution equations for $m, r$ and $\phi$

$$
\begin{aligned}
& 2 \frac{\partial r}{\partial u} \frac{\partial m}{\partial u}=\left(1-\frac{2 m}{r}\right) \frac{r^{2}}{4}\left(\frac{\partial \phi}{\partial u}\right)^{2} \\
& 2 \frac{\partial r}{\partial v} \frac{\partial m}{\partial v}=\left(1-\frac{2 m}{r}\right) \frac{r^{2}}{4}\left(\frac{\partial \phi}{\partial v}\right)^{2} \\
& r \frac{\partial^{2} r}{\partial u \partial v}=\frac{2 m}{r-2 m} \frac{\partial r}{\partial u} \frac{\partial r}{\partial v} \\
& r \frac{\partial^{2} \phi}{\partial u \partial v}+\frac{\partial r}{\partial u} \frac{\partial \phi}{\partial v}+\frac{\partial r}{\partial v} \frac{\partial \phi}{\partial u}=0
\end{aligned}
$$

The quantity

$$
\mu(u, v) \equiv \frac{2 m(u, v)}{r}
$$

plays a crucial rôle in the problem. If $\mu$ stays everywhere below 1 , the field configuration will not collapse but will finally disperse at infinity as outgoing waves. By contrast, if the mass ratio $\mu$ can reach anywhere the value 1 , this signals the formation of an apparent horizon $\mathcal{A}$. The location of this apparent horizon is indeed defined by the equation

$$
\mathcal{A}: \quad \mu(u, v)=1 \text {. }
$$

The above statements are substantiated by some rigorous inequalities [38] stating that:

$$
\begin{array}{ll}
\frac{\partial r}{\partial u}<0, & \frac{\partial m}{\partial v}>0 \\
\frac{\partial r}{\partial v}(1-\mu)>0, & \frac{\partial m}{\partial u}(1-\mu)<0 .
\end{array}
$$


Thus, in weak-field regions $(\mu<1), \partial_{v} r>0$, while, as $\mu>1, \partial_{v} r<0$, meaning that the outgoing radial null rays ("photons") emitted by the sphere $r=$ const become convergent, instead of having their usual behaviour. This is nothing else but the signature of a CTS!

In the case of spherical symmetry, it has been possible to prove [37 that the presence of trapped surfaces implies the existence of a future singular boundary $\mathcal{B}$ of space-time where a curvature singularity occurs. Furthermore, the behaviour of various fields near the singularity is just that of a quasi-homogeneous DDI as described by Eqs. (8)! This highly non-trivial result strongly supports the idea that PBB inflation in the string frame is the counterpart of gravitational collapse in the Einstein frame.

Reference [37] gives the following sufficient criterion on the strength of characteristic data, considered at some finite retarded time $u$

$$
\frac{2 \Delta m}{\Delta r} \geq\left[\frac{r_{1}}{r_{2}} \log \left(\frac{r_{1}}{2 \Delta r}\right)+\frac{6 r_{1}}{r_{2}}-1\right]
$$

where $r_{1} \leq r_{2}$, with $r_{2} \leq 3 r_{1} / 2$, are two spheres, $\Delta r=r_{2}-r_{1}$ is the width of the "annular" region between the two spheres, and $\Delta m=m_{2}-m_{1} \equiv m\left(u, r_{2}\right)-m\left(u, r_{1}\right)$ is the mass "contained" between the two spheres, i.e. more precisely the energy flux through the outgoing null cone $u=$ const, between $r_{1}$ and $r_{2}$. Note the absence of any intrinsic scale (in particular of any short-distance cut-off) in the above criterion. The theorem proved in [37] is not exhausted in the above statement. It contains various bounds as well, e.g.

- an upper bound on the retarded time at which the CTS (i.e. a horizon) is formed,

- a lower bound on the mass, i.e. on the radius of the collapsing region.

The latter quantity is very important for the discussion of the previous subsection since it gives, in the equivalent string-frame problem, an upper limit on the Hubble parameter at the beginning of DDI. Such an upper limit depends only on the size of the advanced-time interval satisfying the $\mathrm{CC}$; since the latter is determined by the scale-invariant condition (30), the initial scale of inflation will be classically undetermined.

The above criterion is rigorous but probably too conservative. It also has the shortcoming that it cannot be used directly on $\mathcal{I}^{-}$, since $u \rightarrow-\infty$ on $\mathcal{I}^{-}$. In Ref. [31] a less rigorous (or less general) but simpler criterion directly expressible in terms of the News (i.e. on $\mathcal{I}^{-}$) was proposed on the basis of a perturbative study. It has the following attractive form:

$$
\sup _{\substack{v_{1}, v_{2} \\ v_{1} \leq v_{2}}} \operatorname{Var}(N(x))_{x \in\left[v_{1}, v_{2}\right]}>C=O(1 / 4)
$$

where:

$$
\operatorname{Var}(N(x))_{x \in\left[v_{1}, v_{2}\right]} \equiv\left\langle\left(N(x)-\langle N\rangle_{\left[v_{1}, v_{2}\right]}\right)^{2}\right\rangle_{x \in\left[v_{1}, v_{2}\right]} .
$$

Thus $\operatorname{Var}(g)_{\left[v_{1}, v_{2}\right]}$ denotes the "variance" of the function $g(x)$ over the interval $\left[v_{1}, v_{2}\right]$, i.e. the average squared deviation from its mean value. 
According to this criterion the largest interval satisfying (31) determines the size of the collapsing region and thus, through the collapse inflation connection, the initial value of the Hubble parameter. It would be interesting to confirm the validity of the above criterion and to determine more precisely the value of the constant appearing on its r.h.s. through more analytic or numerical work. Actually, numerical studies of spherically symmetric PBB cosmologies have already appeared [39], while more powerful numerical codes should soon be available [40].

\section{Phenomenological Consequences}

\subsection{Cosmological amplification of vacuum fluctuations: general prop- erties}

I will start by recalling the basic physical mechanism underlying particle production in cosmology (for a nice review, see [41]) and by introducing the corresponding (and by now standard) jargon. By the very definition of inflation $(\ddot{a}>0)$ physical wavelengths are stretched past the Hubble scale $\left(\mathrm{H}^{-1}\right)$ during inflation. After the end of inflation each wavelength grows slower than $H^{-1}$ and thus "re-enters" the horizon. Obviously, the larger the scale the earlier it crosses the horizon outward and the later it crosses it back inward. Hence larger scales "spend" more time "outside the horizon" than smaller ones.

The attentive reader may worry at this point about the way this description applies when distances are measured using the Einsten-frame metric. As we have seen in the previous section, PBB inflation corresponds to accelerated contraction in the Einstein frame. Nonetheless, one can show that physical quantities (that is, typically, dimensionless ratios of physical quantities) do not depend on the choice of the frame: after all, changing frame is nothing more than a local field-redefinition, which is known not to affect the physics. It is amusing to notice, for instance, that physical wavelengths go outside the horizon during the Einstein-frame equivalent of DDI. Indeed, although physical EF scales shrink during the collapse, the horizon $H^{-1}$ shrinks even faster! I refer to the first paper in [16] for further discussion on this point.

Consider now a generic perturbation $\Psi$ on top of a homogeneous background, which includes a cosmological-type metric, a dilaton, and, possibly, other fields, such as another inflaton field, an axion, etc. Since $\Psi=0$ is, by definition of a perturbation, a classical solution, $\Psi$ intself enters the effective low-energy action quadratically. Soon after the beginning of inflation the background itself becomes homogeneous, isotropic, and spatially flat, so that the perturbed action takes the generic form:

$$
I=\frac{1}{2} \int d \eta d^{3} x S(\eta)\left[\Psi^{\prime 2}-(\nabla \Psi)^{2}\right] .
$$

Here $\eta$ is conformal-time $(a d \eta=d t)$, and a prime denotes $\partial / \partial \eta$. The function $S(\eta)$ (sometimes called the "pump" field) is, for any given $\Psi$, a given function of the scale factor $a(\eta)$, and of other scalar fields (four-dimensional dilaton $\phi(\eta)$, moduli $b_{i}(\eta)$, etc.), which may appear non-trivially in the background. 
While it is clear that a constant $S$ may be reabsorbed by rescaling $\Psi$, and is thus ineffective, a time-dependent $S$ couples non-trivially to $\Psi$ and leads to the production of pairs of quanta (with equal and opposite momenta). In order to see this, it is useful to go over to a Hamiltonian description of the perturbation and of its canonically conjugate momentum $\Pi$ :

$$
\Pi=\frac{\delta I}{\delta \Psi^{\prime}}=S \Psi^{\prime} .
$$

The Hamiltonian corresponding to (33) is thus given by

$$
H=\frac{1}{2} \int d^{3} x\left[S^{-1} \Pi^{2}+S(\nabla \Psi)^{2}\right],
$$

and the first-order Hamilton equations read

$$
\Psi^{\prime}=\frac{\delta H}{\delta \Pi}=S^{-1} \Pi, \quad \Pi^{\prime}=-\frac{\delta H}{\delta \Psi}=S \nabla^{2} \Psi
$$

leading to the decoupled second order equations

$$
\Psi^{\prime \prime}+\frac{S^{\prime}}{S} \Psi^{\prime}-\nabla^{2} \Psi=0, \quad \Pi^{\prime \prime}-\frac{S^{\prime}}{S} \Pi^{\prime}-\nabla^{2} \Pi=0 .
$$

In Fourier space the Hamiltonian (35) is given by

$$
H=\frac{1}{2} \sum_{\vec{k}}\left(S^{-1} \Pi_{\vec{k}} \Pi_{-\vec{k}}+S k^{2} \Psi_{\vec{k}} \Psi_{-\vec{k}}\right),
$$

where $\Psi_{-\vec{k}}=\Psi_{\vec{k}}^{*}$ and $\Pi_{-\vec{k}}=\Pi_{\vec{k}}^{*}$. The equations of motion become

$$
\Psi_{\vec{k}}^{\prime}=S^{-1} \Pi_{-\vec{k}}, \quad \quad \Pi_{\vec{k}}^{\prime}=-S k^{2} \Psi_{-\vec{k}},
$$

where $k=|\vec{k}|$. The transformation

$$
\Pi_{\vec{k}} \rightarrow \widetilde{\Pi}_{\vec{k}}=k \Psi_{\vec{k}}, \quad \Psi_{\vec{k}} \rightarrow \widetilde{\Psi}_{\vec{k}}=-k^{-1} \Pi_{\vec{k}}, \quad S \rightarrow \widetilde{S}=S^{-1}
$$

leaves the Hamiltonian, Poisson brackets, and equations of motion unchanged. This symmetry of linear perturbation theory, and its physical consequences, was discussed in [42 under the name of S-duality, since it contains the usual strong-weak coupling (electric-magnetic) duality in the special case of gauge perturbations.

In order to solve the perturbation equations, and to normalize the spectrum, it is convenient to introduce the normalized (but no longer canonically conjugate) variables $\widehat{\Psi}, \widehat{\Pi}$, whose Fourier modes are defined by

$$
\widehat{\Psi}_{k}=S^{1 / 2} \Psi_{k}, \quad \widehat{\Pi}_{k}=S^{-1 / 2} \Pi_{k},
$$

so that the Hamiltonian density takes the canonical form:

$$
H=\frac{1}{2} \sum_{\vec{k}}\left(\left|\widehat{\Pi}_{k}\right|^{2}+k^{2}\left|\widehat{\Psi}_{k}\right|^{2}\right) .
$$


Under S-duality, these new variables transform as the original ones. They satisfy the Schrödingerlike equations

$$
\widehat{\Psi}_{k}{ }^{\prime \prime}+\left[k^{2}-\left(S^{1 / 2}\right)^{\prime \prime} S^{-1 / 2}\right] \widehat{\Psi}_{k}=0, \quad \widehat{\Pi}_{k}{ }^{\prime \prime}+\left[k^{2}-\left(S^{-1 / 2}\right)^{\prime \prime} S^{1 / 2}\right] \widehat{\Pi}_{k}=0 .
$$

The amplification of perturbations is typically associated with a transition from an inflationary phase in which the pump field is accelerated to a post-inflationary phase in which the pump field is decelerated or constant. In such a class of backgrounds, the "effective potentials", $V_{\Psi}=\left(S^{1 / 2}\right)^{\prime \prime} S^{-1 / 2}$ and $V_{\Pi}=\left(S^{-1 / 2}\right)^{\prime \prime} S^{1 / 2}$, grow during the phase of accelerated evolution, and decrease in the post-inflationary, decelerated epoch, vanishing asymptotically both for very early times, $\eta \rightarrow-\infty$, and for very late times, $\eta \rightarrow+\infty$.

The initial evolution of perturbations, for all modes with $k^{2}>\left|V_{\Psi}\right|,\left|V_{\Pi}\right|$, may be described by the WKB-like approximate solutions of Eqs. (43)

$$
\begin{aligned}
& \widehat{\Psi}_{k}(\eta)=\left(k^{2}-V_{\Psi}\right)^{-1 / 4} e^{-i \int_{\eta_{0}}^{\eta} d \eta^{\prime}\left(k^{2}-V_{\Psi}\right)^{1 / 2}}, \\
& \widehat{\Pi}_{k}(\eta)=k\left(k^{2}-V_{\Pi}\right)^{-1 / 4} e^{-i \int_{\eta_{0}}^{\eta} d \eta^{\prime}\left(k^{2}-V_{\Pi}\right)^{1 / 2}},
\end{aligned}
$$

which we have normalized to a vacuum fluctuation, and where the extra factor of $k$ in the solution for $\widehat{\Pi}_{k}$ comes from consistency with the first order equations (39). We have ignored a possible relative phase in the solutions. Solutions (44) manifestly preserve the S-duality symmetry of the equations, since the potentials $V_{\Psi}, V_{\Pi}$ get interchanged under $S \rightarrow S^{-1}$.

Let us now discuss two opposite regimes:

- When the perturbation is deeply inside the horizon $(k / a \gg H)$ we find "adiabatic" behaviour, i.e.

$$
k \Phi_{k} \sim S^{-1 / 2}, \Pi_{k} \sim S^{1 / 2},
$$

implying, through (35), that the contribution to the Hamiltonian of modes inside the horizon stays constant.

- When the perturbation is far outside the horizon $(k / a \ll H)$, it enters the so-called freeze-out regime in which $\Psi$ and $\Pi$ stay constant (better have a constant solution, see [42]). Such a behaviour implies, again through (35), that the contribution of super-horizon modes to the Hamiltonian grows in time. If $\dot{S}>0$, the growth of $\mathcal{H}$ is due to $\Psi$, while, for $\dot{S}<0$, it is due to $\Pi$. In either case the growth is due to particle production in squeezed states [43], i.e. states in which one canonical variable is very sharply defined and the conjugate one is largely undetermined. Although, strictly speaking, quantum coherence is not lost, in practice the sub-fluctuating variable cannot be measured with unlimited precision (coarse graining) and therefore entropy is produced (see Subsection 4.6).

It is not too hard to join the two extreme regimes mentioned above and to find the qualitative and quantitative features of the solutions. For lack of space we refer the reader to the original literature (see, e.g. [42]). 
The above considerations were very general. What is instead typical of the PBB scenario? There are at least two features that are quite unique to string cosmology:

- Pump fields, and in particular their contributions to the evolution equations (43), grow during PBB inflation, while they tend to decay in standard inflation.

- The richer set of backgrounds and fluctuation present in string theory allows for the amplification of new kinds of perturbations.

One can easily determine the pump fields for each one of the most interesting perturbations appearing in the PBB scenario. The result is:

$$
\begin{aligned}
\text { Gravity waves, dilaton } & : S=a^{2} e^{-\phi} \\
\text { Heterotic gauge bosons } & : S=e^{-\phi} \\
\text { Kalb - - Ramond, axions } & : S=a^{-2} e^{-\phi} .
\end{aligned}
$$

In the following subsections we shall briefly describe the characteristics of these four perturbations after their original vacuum fluctuations are amplified by PBB inflation. For further details, see also [44].

\subsection{Tensor perturbations: an observable cosmic gravitational radi- ation background (CGRB)?}

It is not surpising to find that, for tensor and dilaton perturbations, the pump field is nothing but the scale factor in the Einstein frame $\left(a_{E}=a e^{-\phi / 2}\right)$ since, in this frame, the action for gravity and for the dilaton take the canonical form. The Einstein-frame scale factor corresponds to a collapsing Universe (see Section 3), hence to the decreasing pump field $a_{E}(\eta) \sim \eta^{1 / 2}$ during DDI. For scales that go outside the horizon during DDI, this implies [45 a Raileigh-Jeans-like spectrum, $d \Omega / d \log k \sim k^{3}$, up to logarithmic corrections 45 .

When the curvature scale reaches the string scale we expect DDI to end, and a high (string scale) curvature phase to follow, before the eventual exit to the FRW phase takes place (see Section 5). Not much is known about the string phase, but, using some physical arguments as well as some quantitative estimates, it can be argued that such a phase will lead to copious GW production at frequencies corresponding to the string scale at the time of exit. After the transition to the FRW phase, all particle production switches off. This is why our GW spectrum has an end point that corresponds to the string/Planck scale at the beginning of the FRW phase. If no inflation takes place after, the end-point frequency corresponds, today, to $\omega=\omega_{1} \sim 100 \mathrm{GHz}$.

As illustrated in Fig. 8, the GW spectrum can be rather flat below the end point, up to the frequency $\omega_{s}$, the last scale that went out of the horizon during DDI. Further below $\omega_{s}$ we get 


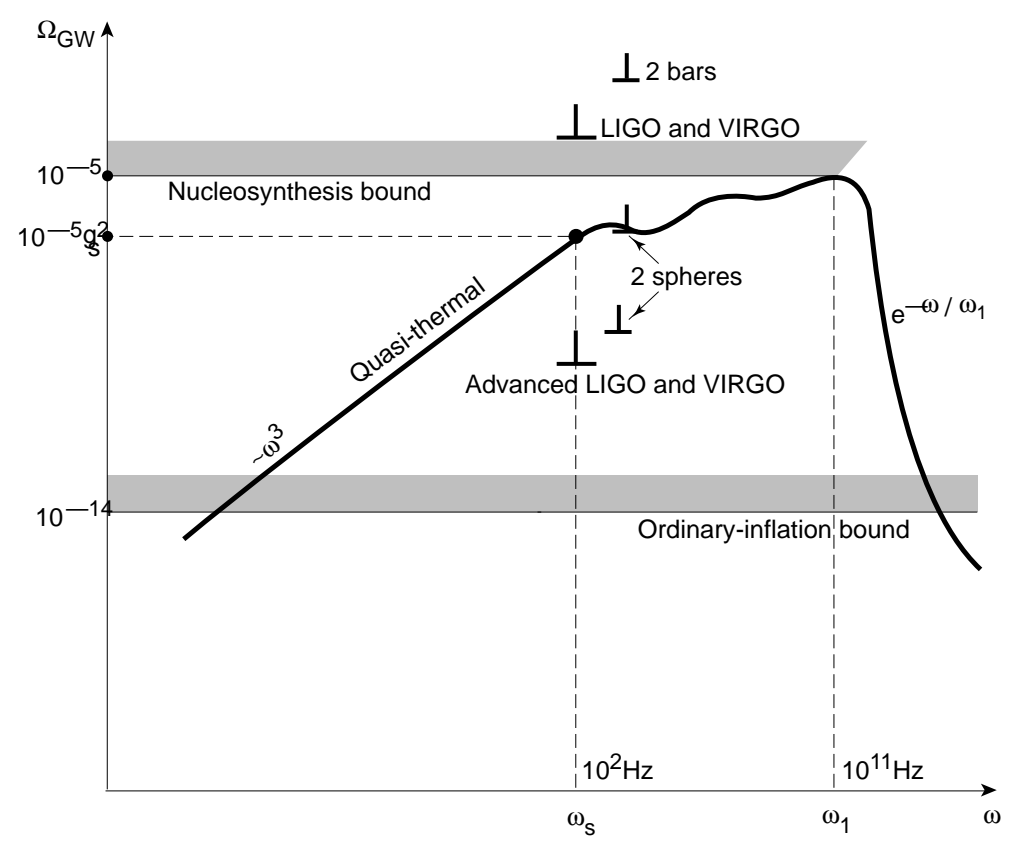

Figure 8

the above-mentioned steep $\omega^{3}$ spectrum. It thus looks as if the best chances for the detection of our stochastic background lie precisely near $\omega_{s}$, where a kink (or knee) is expected.

Unfortunately, the position of the knee and the value of $\Omega_{G W}$ at that point depend on two background parameters that are, so far, difficult to predict. One corresponds to the duration of (better, the total red-shift during) the string phase, the other to the value of $l_{P} / \lambda_{s}$ at the end of DDI (hence to the value of the dilaton at that time). As shown in Fig. 8, values of $\Omega_{G W}$ in the range of $10^{-6}-10^{-7}$ are possible in some regions of parameter space, which, according to some estimates of sensitivities [46] reported in the same figure for $\omega_{s} \sim 10^{2} \mathrm{~Hz}$, could be inside detection capabilities in the near future.

The signal is predicted to consist of randomly distributed standing waves, a feature that has been argued [47] to further help detection. In any case, cross-correlation experiments are mandatory here in order to disentangle this stochastic signal from real noise. Sensitivities to a CGRB of this type have been estimated for a variety of two-detector combinations [46]. A comprehensive review of GW experiments and of their relevance to the early Universe can be found in 48 .

\subsection{Dilaton perturbations}

Since the dilaton is, after all, the inflaton of PBB cosmology, its fluctuations are the most natural source of adiabatic scalar perturbations. We recall that, in standard cosmology, inflaton fluctuations naturally lead to a quasi scale-invariant, Harrison-Zeldovich (HZ) spectrum 
of adiabatic perturbations, something highly desirable both to explain CMB anisotropy and for models of LSS formation. Can we get something similar from the dilaton? The answer, unfortunately, is no! Let me spend a moment explaining why.

Unlike tensor perturbations, which do not couple to the scalar field to linear order and are gauge-invariant by themselves, scalar perturbations are contained, a priori, in five functions defined by:

$$
\begin{aligned}
d s^{2} & =a^{2}(\eta)\left[-(1+2 \Phi) d \eta^{2}+\left((1-2 \Psi) \delta_{i j}+\partial_{i} \partial_{j} E\right) d x^{i} d x^{j}-2 \partial_{i} B d x^{i} d \eta\right] \\
\phi & =\phi_{0}(\eta)+\chi(\eta, \vec{x}) .
\end{aligned}
$$

The five functions $\Phi, \Psi, B, E, \chi$ are not separately gauge-invariant. However, the following "Bardeen" combinations are gauge-invariant:

$$
\begin{aligned}
\Phi_{B} & =\Phi+\frac{1}{a}\left[a\left(B-E^{\prime}\right)\right]^{\prime}, \\
\Psi_{B} & =\Psi-\frac{a^{\prime}}{a}\left(B-E^{\prime}\right), \\
\chi_{G I} & =\chi+\frac{\phi_{0}^{\prime} a}{a^{\prime}} \Psi .
\end{aligned}
$$

Introducing the variable $v \equiv a \chi_{G I}$, the scalar field enters the quadratic action "canonically" i.e.:

$$
S_{e f f}(v)=\frac{1}{2} \int d \eta d^{3} x\left[v^{\prime 2}-(\vec{\nabla} v)^{2}+\left(z^{\prime \prime} / z\right) v^{2}\right], \quad z \equiv \frac{\phi_{0}^{\prime} a^{2}}{a^{\prime}}
$$

giving the evolution equation

$$
v_{k}^{\prime \prime}+\left(k^{2}-\left(z^{\prime \prime} / z\right)\right) v_{k}=0
$$

In the DDI background, $z \sim a$ and thus the canonical scalar field obeys the same equation as the canonical graviton field, therefore giving identical spectra (as far as the dilaton remains massless, of course). This strongly suggests that adiabatic perturbations in PBB cosmology have a Raleigh-Jeans, rather than HZ, spectrum and that they are unsuitable for generating CMBA or LSS. Before being sure of that, however, we have to analyse the scalar fluctuations of the metric itself in terms of the above-mentioned Bardeen potentials $\Phi_{B}, \Psi_{B}$.

A popular gauge (particularly advertised in [41) is the so-called longitudinal gauge, defined by $B=E=0$, where $\Phi_{B}=\Phi$ and $\Psi_{B}=\Psi$. In this gauge one of the constraints simply reads $\Phi=\Psi$, while a second constraint relates either one of them to $v$ :

$$
\Psi_{k}=-\frac{\phi_{0}^{\prime}}{4 k^{2}}\left(v_{k} / a\right)^{\prime} \sim k^{-3 / 2} \frac{1}{|k \eta|^{2}}(k / a)_{H C},
$$

where we have inserted the small $k$ behaviour of $v_{k}$, which is identical to that of tensor perturbations.

Unfortunately, Eq. (51) leads to very large fluctuations of $\Psi=\Phi$ at small $k \eta$, so large that one leaves the linear-perturbation regime for the expansion (47) of the metric much before the 
high-curvature scale is reached. Does this mean that the metric becomes very inhomogeneous? It would look to be the case ... unless the growth of $\Psi$ and $\Phi$ is in some way a gauge artefact. But how can it be a gauge artefact if $\Psi$ and $\Phi$ correspond, in this gauge, to the gauge-invariant Bardeen potentials? The answer to this question was provided in [49]. By going from the longitudinal gauge to an "off-diagonal" gauge with $\Psi=E=0$, or, even better, to one in which only $\Phi$ and $E$ appear, one finds that perturbations of the metric remain small at all $\eta$ till Planckian/string-scale curvatures are reached.

This is easy to see, for instance, in a gauge with $\Psi=B=0$, where $\Psi_{B} \sim\left(a^{\prime} / a\right) E^{\prime}$. Clearly this gives $E \sim \eta^{2} \Psi_{B}$ and, since $E$ enters the metric with two spatial derivatives, this implies that

$h_{i j} \sim(k \eta)^{2} \Psi_{B}$, which is sufficiently small at small $k \eta$ for linear perturbation theory to be valid. One can then look for physical effects of these scalar perturbations (e.g. for contributions to CMBA) and find that they actually remain as small as the tensor contributions. In conclusion, once gauge artefacts are removed, it seems that adiabatic scalar perturbations, as well as their tensor counterparts, remain exceedingly small at large scales.

On the other hand, the rather large yields at short scales also apply to dilatons. This allows for a possible source of scalar waves if the dilaton is very light. However, as recently discussed by Gasperini [50], it is very unlikely that such a signal will be observable, given the constraints on the dilaton mass due to tests of the equivalence principle (see Section 2). Other restrictions on the dilaton mass come from the possibility that their density may become overcritical and close the Universe. This and other possible interesting windows in parameter space are discussed in [17], and will not be reported in any detail here.

\subsection{Gauge-field perturbations: seeds for $\vec{B}_{g a l}$ ?}

In standard inflationary cosmology there is no amplification of the vacuum fluctuations of gauge fields. This is a straightforward consequence of the fact that inflation makes the metric conformally flat, and of the decoupling of gauge fields from a conformally flat metric precisely in $D=3+1$ dimensions.

As a very general remark, apart from pathological solutions, the only background field that can amplify, through its cosmological variation, e.m. (more generally gauge-field) quantum fluctuations is the effective gauge coupling itself [51]. By its very nature, in the pre-big bang scenario the effective gauge coupling inflates together with space during the PBB phase. It is thus automatic that any efficient PBB inflation brings together a huge variation of the effective gauge coupling, and thus a very large amplification of the primordial e.m. fluctuations [52]. This can possibly provide the long-sought for origin for the primordial seeds of the observed galactic magnetic fields.

To be more quantitative, since the pump field for electromagnetic perturbations is the effective (four-dimensional) gauge coupling itself (see Eq. (46)), the total amplification of e.m. perturbations on any given scale $\lambda$ is given by $\alpha_{0} / \alpha_{e x}$, i.e. by the ratio of the fine structure constant now and the fine structure constant at the time of exit of the scale $\lambda$ during DDI. It 
turns out [52 that, in order to produce sufficiently large seeds for the galactic magnetic fields, such a ratio has to be enormous for the galactic scale, i.e. about $10^{66}$. Taken at face value, this would be a very strong indication in favour of the PBB scenario, more particularly of DDI. Indeed, only in such a framework is it natural to expect that the effective gauge coupling grew during inflation by a factor whose logarithm is of the same order as the number of inflationary e-folds.

Notice, however, that, unlike GW, e.m. perturbations interact quite considerably with the hot plasma of the early (post-big bang) Universe. Thus, converting the primordial seeds into those that may have existed at the protogalaxy formation epoch is by no means a trivial exercise (see, e.g. [53]). The question of whether or not the primordial seeds generated in PBB cosmology can evolve into the observed galactic magnetic fields thus remains, to this date, an unsolved, yet very interesting, problem.

\subsection{Axion perturbations: seeds for CMBA and LSS?}

In four dimensions the curl of $B_{\mu \nu}, H_{\mu \nu \rho}$, is equivalent to a pseudoscalar field, the (KR) axion $\sigma$, through

$$
H_{\mu \nu \rho}=e^{\phi} \epsilon_{\mu \nu \rho \tau} \partial^{\tau} \sigma .
$$

It is easy to see that, while the pump field for $B_{\mu \nu}$ is $a^{-2} e^{-\phi}$, that for $\sigma$ is $a^{2} e^{\phi}$. Indeed their respective perturbations are related by the duality of perturbations discussed in Subsection 4.1. We can use either description with identical physical results. Note that, while $a$ and $\phi$ worked in opposite directions for tensor and dilaton perturbations, generating strongly tilted (blue) spectra, the two work in the same direction for axions, so that spectra can be flat or even tilted towards large scales (red spectra) [54]. An interesting fact is that, unlike the GW spectrum, that of axions is very sensitive to the cosmological behaviour of internal dimensions during the DDI epoch. On one side, this makes the model less predictive. On the other, it tells us that axions represent a window over the multidimensional cosmology expected generically from string theories, which must live in more that four dimensions. Parametrizing the spectrum by:

$$
\Omega_{a x}(k)=\left(\frac{H_{\max }}{M_{P}}\right)^{2}\left(k / k_{\max }\right)^{\alpha}
$$

and considering the case of three non-compact and six compact dimensions with separate isotropic evolution, one finds:

$$
\alpha=\frac{3+3 r^{2}-2 \sqrt{3+6 r^{2}}}{1+3 r^{2}}
$$

where

$$
r \equiv \frac{1}{2} \frac{\dot{V}_{6} V_{3}}{V_{6} \dot{V}_{3}}
$$

is a measure of the relative evolution of the internal and external volumes. Equations (54), (55) show that the axion spectrum becomes exactly $\mathrm{HZ}$ (i.e. scale-invariant) when $r=1$, i.e. 
when all nine spatial dimensions of superstring theory evolve in a rather symmetric way [55]. In situations near this particularly symmetric one, axions are able to provide a new mechanism for generating large-scale CMBA and LSS.

Calculation of the effect gives [56], for massless axions:

$$
l(l+1) C_{l} \sim O(1)\left(\frac{H_{\max }}{M_{P}}\right)^{4}\left(\eta_{0} k_{\max }\right)^{-2 \alpha} \frac{\Gamma(l+\alpha)}{\Gamma(l-\alpha)},
$$

where $C_{l}$ are the usual coefficients of the multipole expansion of $\Delta T / T$

$$
\left\langle\Delta T / T(\vec{n}) \quad \Delta T / T\left(\vec{n}^{\prime}\right)\right\rangle=\sum_{l}(2 l+1) C_{l} P_{l}(\cos \theta), \quad \vec{n} \cdot \vec{n}^{\prime}=\cos \theta,
$$

and $\eta_{0} k_{\text {max }} \sim 10^{30}$. In string theory, as repeatedly mentioned, we expect $H_{\text {max }} / M_{P} \sim M_{s} / M_{P} \sim$ $1 / 10$, while the exponent $\alpha$ depends on the explicit PBB background with the above-mentioned HZ case corresponding to $\alpha=0$. The standard tilt parameter $n=n_{s}$ ( $s$ for scalar) is given by $n=1+2 \alpha$ and is found, by COBE[57], to lie between 0.9 and 1.5, corresponding to $0<\alpha<0.25$ (a negative $\alpha$ leads to some theoretical problems). With these inputs we can see that the correct normalization $\left(C_{2} \sim 10^{-10}\right)$ is reached for $\alpha \sim 0.2$, which is just in the middle of the allowed range. In other words, unlike in standard inflation, we cannot predict the tilt, but when this is given, we can predict (again unlike in standard inflation) the normalization.

With some extra work [58] one can compute the $C_{l}$ in the acoustic-peak region adding vector

and tensor contributions from the seeds. It turns out that the acoustic-peak structure is very sensitive to $\alpha$, hence to the behaviour of the internal dimensions during the DDI phase. The above-mentioned value, $\alpha=1$, does not give peaks at all and, as such, looks ruled out by the data. Values of $\alpha$ in the range $0.3-0.4$ appear to be preferred (especially in the presence of a cosmological constant with $\Omega_{\Lambda} \sim 0.7$ ). We saw, however, that the overall normalization was very sensitive to the value of $\alpha$. For $\alpha$ in the $0.3-0.4$ range, the normalization is off (way too small) by many orders of magnitude. Therefore, if present indications are confirmed, as they seem to be from the recent release of the Boomerang 1997 data analysis [59], one will be forced to a $k$-dependent $\alpha$, meaning different phases in the evolution of internal dimensions during DDI.

\subsection{Heating up the Universe}

Before closing this section, I wish to recall how one sees the very origin of the hot big bang in this scenario. One can easily estimate the total energy stored in the quantum fluctuations, which were amplified by the pre-big bang backgrounds (for a discussion of generic perturbation spectra, see [55, 60]. The result is, roughly,

$$
\rho_{\text {quantum }} \sim N_{\text {eff }} H_{\text {max }}^{4},
$$

where $N_{\text {eff }}$ is the effective number of species that are amplified and $H_{\max }$ is the maximal curvature scale reached around $t=0$. We have already argued that $H_{\text {max }} \sim M_{s}=\lambda_{s}^{-1}$, and 
we know that, in heterotic string theory, $N_{\text {eff }}$ is in the hundreds. Yet, this rather huge energy density is very far from critical, as long as the dilaton is still in the weak-coupling region, justifying our neglect of back-reaction effects. It is very tempting to assume [55] that, precisely when the dilaton reaches a value such that $\rho_{\text {quantum }}$ is critical, the Universe will enter the radiation-dominated phase. This PBBB (PBB bootstrap) constraint gives, typically:

$$
e^{\phi_{e x i t}} \sim 1 / N_{\text {eff }}
$$

i.e. a value for the dilaton close to its present value.

The entropy in these quantum fluctuations can also be estimated following some general results [61]. The result for the density of entropy $S$ is, as expected,

$$
S \sim N_{\text {eff }} H_{\max }^{3}
$$

It is easy to check that, at the assumed time of exit given by (59), this entropy saturates recently proposed holography bounds. The discussion of such bounds is postponed to Subsection 5.7 since is has also interesting implications for the exit problem.

\section{$5 \quad$ How could it have stopped?}

We have argued that, generically, DDI, when considered at lowest order in derivatives and coupling, evolves towards a singularity of the big bang type. Similarly, at the same level of approximation, non-inflationary solutions of the FRW type emerge from a singularity. Matching these two branches in a smooth, non-singular way has become known as the (graceful) exit problem in string cosmology [62]. It is, undoubtedly, the most important theoretical problem the $\mathrm{PBB}$ scenario is facing today.

Of course, one would not only like to know that a graceful exit does take place: one would also like to describe the transition between the two phases in a quantitative way. Achieving this goal would amount to nothing less than a full description of what replaces the big bang of standard cosmology in the PBB scenario. As mentioned in Section 1, this difficult problem is the analogue, in string cosmology, of the (still not fully solved) confinement problem of QCD. The exit problem is particularly hard because, by its very nature, and by the existing no-go theorems [62], it must occur, if at all, at large curvature and/or coupling and, because of fast time-dependence, must break (spontaneously) supersymmetry. The phenomenological predictions made in the previous section were based on the assumption that i) a graceful exit does take place; ii) sufficiently large scales are only affected by it kinematically, i.e. through an overall red-shift of all scales.

In this section, after recalling some no-go theorems for the exit, we will review various proposals that circumvent those theorems starting from the mathematically simplest, but physically least realistic, proposals and ending with the physically favoured, but harder to analyse, suggestions. The latter proposals suggest possible lines along which a quantitative description of the exit might eventually emerge. Needless to say, in spite of the many encouraging results, 
much work remains to be done: perhaps new techniques, and/or a deeper understanding of string theory in its non-perturbative regimes through the construction of the still largely unknown M-theory [10, need to be developed before a full quantitative description can be hoped for.

I should also mention that there have been suggestions [63 that the BB singularity can be avoided if the DDI phase is highly anisotropic. While this is an interesting suggestion, with isotropization taking place later in the non-inflationary regime, we will stick here to the simplest case, in which DDI has already prepared a very homogeneous Universe before exit takes place. This is why our discussion on the exit problem is limited to the case of homogeneous cosmologies. Also, for lack of space, I shall refer the reader to the literature for most of the details.

\section{$5.1 \quad$ No-go theorems}

Under some restrictive conditions [62], it was shown that one cannot have a change of branch, i.e. that the Universe cannot make a permanent transition from the inflationary pre-big bang to a FRW post-big bang solution. Perhaps the best way to convey the physical meaning of those theorems is in terms of the necessary conditions for exit recently given by Brustein and Madden 64]. These authors give necessary conditions for two subsequent events to occur: firstly, a branch change in the string frame should take place: this imposes the violation of some energy conditions; secondly, a bounce should occur in the E-frame metric since, as we have seen in Section 3, DDI represents a collapse in the E-frame. This latter transition requires further violation of energy conditions.

Before the reader gets too worried about these violations, I should point out that these refer to the equations of state satisfied by some "effective" sources, which include both higherderivative and higher-loop corrections. It is well known that such sources generically do lead to violations of the standard energy conditions satisfied by normal matter or radiation-like classical sources.

\subsection{Exit via a non-local $V$}

This is perhaps the simplest example of an exit. It was first discussed in [65]. The reason why this is not considered an appealing mechanism for the exit is that the potential it employs depends on $\bar{\phi}$ (instead of $\phi$ ), in order to preserve SFD. By general covariance such a potential, if non-trivial, must be non-local. Unfortunately, there has been no convincing proposal to explain how such non-local potentials might arise within a superstring theory framework. 


\section{$5.3 \quad$ Exit via $B_{i j}$}

The antisymmetric KR field may lead to violation of the energy conditions and thus induce an exit. Some amusing examples were given in [66], where a non-trivial $B_{i j}$ is introduced through $O(d, d)$ transformations acting on a pure metric-dilaton cosmology of the type described in Subsection 2.5. It was found that these so-called "boosted" cosmologies were less singular than the original ones. In some cases they were even completely free of singularity and provided examples of exit, albeit in not-so-realistic situations.

It is tempting to speculate that this softening of singularities, due to a non-trivial $B_{i j}$ field, could be related to recent developments in the field of non-commutative geometry [67] induced by a $B_{i j}$ field. Work along these lines is in progress.

\subsection{Exit via quantum tunnelling}

Several groups [68 have attempted to describe the transition from the pre- to the post-big bang without modifying the low-energy tree-level effective action, by exploiting the quantum cosmology approach based on the Wheeler-De Witt (WDW) equation. In Refs. [68] an $O(d, d)$ invariant WDW equation was derived in the $\left(d^{2}+1\right)$-dimensional mini-superspace consisting of a homogeneous Bianchi I metric, the antisymmetric tensor, and the dilaton. The $O(d, d)$ symmetry helps avoiding the ordering ambiguities which usually plague the WDW equation. For the time being only the mathematically simpler case of an $O(d, d)$-invariant potential $V(\bar{\phi})$ has been analysed since, in that case, $d^{2}$-conserved charges can be defined and the "radial" part of the WDW equation reduces to a one-dimensional Schroedinger equation for a scattering problem.

It is amusing that, from such a point of view, the initial state of the Universe is described by a right-moving plane wave, which later encounters a potential, giving rise to both a transmitted and a reflected (i.e. left-moving) wave. The transmission coefficient gives the probability that the Universe ends up in the pre-big-bang singularity, while the reflection coefficient gives the probability of a successful exit into the post-big-bang decelerating expansion.

For certain forms of $V(\bar{\phi})$ the wave is classically reflected and the WDW approach just confirms this expectation by giving a $100 \%$ probability for the exit. However, even when there is no classical exit, the probability of wave-reflection is non-zero because of quantum tunnelling. The quantum probability of a classically forbidden exit turns out to be exponentially suppressed in the coupling constant $e^{\phi}$, which is just fine. Unfortunately, it is also exponentially suppressed in the total volume of 3-space (in string units) after the pre-big-bang. Thus, only tiny regions of space have a reasonable chance to tunnel. 


\subsection{Higher-derivative corrections}

While the examples of exit given in the previous subsections are theoretically interesting, they do look somewhat artificial and non-generic. In this and in the following subsection we shall describe two mechanisms for exit that involve very general properties of the lowest order solutions and of string theory. The present feeling is that, if graceful exit occurs, it should be maily induced by some combination of higher-derivative and higher-loop effects. Let us start with the former.

Toy examples have shown [69] that DDI can flow, thanks to higher-curvature corrections, towards a de-Sitter-like phase, i.e. into a phase of constant $H$ (curvature) and constant $\dot{\phi}$. This phase is expected to last until loop corrections become important and give rise to a transition to a radiation-dominated phase (see the next subsection). The idea is to justify the strong curvature transition from the dilatonic to the string phase by proving the existence of an exact de Sitter-like solution to the field equation, which acts as a late time attractor for the perturbative DDI branch. As shown in [69], the existence of such attractors depends on the existence of (non-trivial) solutions for a system of $n$ algebraic equations in $n$ unknowns. In general, we may expect a discrete number of solutions to exist. If at least one of them has some qualitative characteristics, it will act as a late-time attractor for solutions approaching DDI in the far past. An explicit example of this phenomenon was constructed in [69]. In this connection, it is worth mentioning that solutions connecting duality-related low-energy branches through a high-curvature CFT were already proposed in [70].

It was recently pointed out [71] that the reverse order of events is also possible. The coupling may become large before the curvature does. In this case, at least for some time, the low-energy limit of M-theory should be adequate: this limit is known [10] to give $D=11$ supergravity and is therefore amenable to reliable study. It is likely, though not yet clear, that, also in this case, strong curvatures will have to be reached before the exit can be completed.

\subsection{Loop corrections and back reaction}

The idea here is to invoke the back reaction from particle production as the relevant mecha-

nism. Since the back reaction is an $O\left(e^{\phi} \alpha^{\prime} H^{2}\right)$ correction, its effect is contained in one-loop $O\left(R^{2}\right)$ contributions to the effective action. A recent calculation [72] shows that, indeed, loop corrections to DDI work in the right direction and become relevant precisely when expected according to the exit criterion (59).

A class of such contributions was analysed some time ago by Antoniadis et al. [73 in the case of a spatially flat $(k=0)$ cosmology and by Easther and Maeda [74 in the case of a closed Universe $(k=1)$. Both groups find non-singular solutions to the loop-corrected field equations. However, neither group is actually able to obtain solutions that start in the dilaton-driven superinflationary regime and later evolve through a branch change.

More recently, several examples of full exit have been constructed [75]. Although they are 
based on $\alpha^{\prime}$ and loop-corrected actions, which have not been derived from reliable calculations, they seem to indicate, at least, that the BM conditions for exit may turn out to be not just necessary but also sufficient. It also appears [76] that exit occurs when the entropy bound becomes threatened by the entropy in the amplified/squeezed quantum fluctuations, as we shall now discuss.

\subsection{Entropy considerations}

Entropy-related considerations have recently led to model-independent arguments in favour of the occurrence of a graceful exit in string cosmology. As we shall see, those are physically quite close to the arguments based on back-reaction and loop corrections, which we have just discussed in the previous subsection.

Almost twenty years ago Bekenstein [77] suggested that, for a limited gravity system of energy $E$, and whose size $R$ is larger than its gravitational radius, $R>R_{g} \equiv 2 G_{N} E$, entropy is bounded by $S_{B E B}$ :

$$
S_{B E B}=E R / \hbar=R_{g} R l_{P}^{-2}
$$

Holography [78 suggests that maximal entropy is bounded by $S_{H O L}$,

$$
S_{H O L}=A l_{P}^{-2}
$$

where $A$ is the area of the space-like surface enclosing the region of space whose entropy we wish to bound. For systems of limited gravity, since $R>R_{g}, A=R^{2}$, (61) implies the holography bound $(62)$.

Can these entropy bounds be applied to the whole Universe, i.e. to cosmology? A cosmological Universe is not a system of limited gravity, since its large-distance behaviour is determined by the gravitational effect of its matter content through Friedmann's equation (4). Furthermore, the holography bound obviously fails for sufficiently large regions of space since, for a given temperature, entropy grows like $R^{3}$ while area grows like $R^{2}$. The generalization of entropy bounds to cosmology turned out to be subtle.

In 1989, Bekenstein himself [79] gave a prescription for a cosmological extension by choosing $R$ in Eq. (61) to be the particle horizon. Amusingly, he arrived at the conclusion that the bound is violated sufficiently near the big-bang singularity, implying that the latter is fake (if the bound is always valid). About a year ago, Fischler and Susskind (FS) [80 proposed a similar extension of the holographic bound to cosmology, arguing that the area of the particle horizon should bound entropy on the backward-looking light cone according to (62). It was soon realized, however, that the FS proposal requires modifications, since violations of it were found to occur in physically reasonable situations. An improvement of the FS bound applicable to light-like hypersurfaces was later made by Bousso [81].

Of more interest here are the attempts made at deriving cosmological entropy bounds on space-like hypersurfaces [82, 83, 84, 85, 86]. These identify the maximal size of a spatial region 
for which holography works: the Hubble radius [82, 83, 85], the apparent horizon [84], or, finally, a so-called causal connection (Jeans) scale [86].

For our purposes here, we do not need to enter into the relative merits of these various proposals. Rather, we will only outline the physical idea behind them. Consider, inside a quasi-homogeneous Universe, a sphere of radius $H^{-1}$. We may consider "isolated" bodies, in the sense of ref. [77], fully contained in the sphere, i.e. with radius $R<H^{-1}$. For such systems, the usual BB holds and is saturated by a black hole of size $R$. We may consider next several black holes inside our Hubble volume, each carrying an entropy proportional to the square of its mass. If two, or more, of these black holes merge, their masses will add up, while the total entropy after the merging, being quadratic in the total mass, will exceed the sum of the initial entropies. In other words, in order to maximize entropy, it pays to form black holes as large as possible.

Is there a limit to this process of entropy increase? The suggestion made in 82, 83, 84, 85, 86], which finds support in old results by several groups [87], is that a critical length of order $H^{-1}$ is the upper limit on how large a classically stable black hole can be. If we accept this hypothesis, the upper bound on the entropy contained in a given region $\mathcal{R}$ of space will be given by the number of Hubble volumes in $\mathcal{R}, n_{H}=V H^{3}$ times the Bekenstein-Hawking entropy of a $\mathrm{BH}$ or radius $H^{-1}, H^{-2} l_{P}^{-2}$. The two factors can be combined in the suggestive formula:

$$
S(\mathcal{R})<l_{P}^{-2} \int_{\mathcal{R}} d^{3} x \sqrt{h} \tilde{H} \equiv S_{H B}
$$

where $\int_{\mathcal{R}} d^{3} x \sqrt{h}$ is the volume of the space-like hypersurface whose entropy we wish to bound, and $\tilde{H}$ differs from one proposal to another [82, 83, 84, 85, 86], but is, roughly, of the order of the Hubble parameter. Actually, since $H$ is proportional to the trace of the second fundamental form on the hypersurface, Eq. (63) reminds us of the boundary term that has to be added to the gravitational action in order to correctly derive Einstein's equations from the usual variational principle. This shows that the bound (63) is generally covariant for $\tilde{H}=H$. It can also be written covariantly for the identification of $\tilde{H}$ made in [86].

For the qualitative discussion that follows, let us simply take $\tilde{H}=H$ and let us convert the bound to string-frame quantities, taking into account the relation between $l_{P}$ and $\lambda_{s}$, given in Eq. (3). We obtain [83]:

$$
S(\mathcal{R})<\left(V H^{3}\right)\left(H^{-2} \lambda_{s}^{-2} e^{-\phi}\right)=e^{-\bar{\phi}} H \lambda_{s}^{-2},
$$

where we have fixed an arbitrary additive constant in the definition (6) of $\bar{\phi}$. Equation (64) thus connects very simply the entropy bound of a region of fixed comoving volume to the most important variables occurring in string cosmology (see, e.g., the phase diagram of Fig. 3).

An immediate application of the bound (64) was pointed out in [83]. Noting that the bound is initially saturated in the BDV picture [31] of collapse/inflation, the bound itself cannot decrease without a violation of the second law. This gives immediately:

$$
\dot{\bar{\phi}} \leq \frac{\dot{H}}{H} .
$$


It is easy to check that this inequality is obeyed, but just so, during DDI, in the sense that it holds with the equality sign. In other words, the HEB is saturated initially and throughout DDI in the BDV picture. The bound also turns out to give a physically acceptable value for the entropy of the Universe just after the big bang: a large entropy on the one hand (about $\left.10^{90}\right)$; a small entropy for the total mass and size of the observable Universe on the other, as often pointed out by Penrose 88]. Thus, PBB cosmology neatly explains why the Universe, at the big bang, looks so fine-tuned (without being so) and provides a natural arrow of time in the direction of higher entropy 83 .

What happens in the mysterious string phase, where we are desperately short of reliable techniques? It is quite clear that Eq. (65) does not allow $H$ to reach saturation $(\dot{H}=0)$ in the first quadrant of Fig. 3 since $\dot{\bar{\phi}}>0$ there. Instead, saturation of $H$ in the second quadrant (where $\dot{\bar{\phi}} \leq 0$ ) is perfectly all right. But this implies having attained the sought for branch change!

Let us finally look at the loop corrections. Since, physically, these correspond to taking into account the back-reaction from particle production, we may check when the entropy in the cosmologically produced particles starts to threaten the bound. As discussed in Subsection 4.6, the entropy density in quantum fluctuations is given by $\sigma \sim N_{\text {eff }} H^{3}$, which equals the bound $\sigma_{H E B} \sim H l_{P}^{-2}$ precisely when $l_{P}^{2} H^{2} N_{e f f}=O(1)$. But, as already pointed out, this is just the line on which the energy density in quantum fluctuations becomes critical (see Eq. (59)) and where, according to [69], the back-reaction becomes $O(1)$. Similar conclusions are reached by applying generalized second law arguments [89.

The picture that finally emerges from all these considerations is best illustrated with reference to the diagram of Fig. 4. Two lines are shown, representing boundaries for the possible evolution. The horizontal boundary is forced upon by the large-curvature corrections, while the tilted line in the first quadrant corresponds to the equation $l_{P}^{2} H^{2} N_{e f f}=O(1)$ that we have just discussed. Amusingly, this line was also suggested by Maggiore and Riotto [71] as a boundary beyond which copious production of 0-branes would set in. Thus, depending on initial conditions, the PBB bubble corresponding to our Universe would hit first either the high-curvature or the large-entropy boundary and initiate an exit phase. Hopefully, a universal late-time attractor will emerge guiding the evolution into the FRW phase of standard cosmology (shown as a vertical line in Fig. 10).

Needless to say, all this has to be considered, at best, as having heuristic value. If taken seriously, it would suggest that the Universe will never enter the strong-coupling, strong-curvature regime, where the largely unknown M-theory should be used. The low-energy limit of the latter (the much better understood 11-D supergravity) could suffice to deal with the fundamental exit problem of string cosmology. We refer to the literature for several other attempts at M-cosmology 90. 


\section{Outlook}

The outlook for the pre-big bang scenario, as formulated at present, is not necessarily an optimistic one. I am not sure I would bet a lot of money on it being right! But this is not really the issue. We have to remember that the PBB scenario is a top-down approach to cosmology. As stressed in the introduction, it would be quite a miracle if the correct model could be guessed without extensive feed-back from the data. The good news here is that new data are coming in all the time, and will continue to do so with more and more precision in the coming years!

Rather, we should draw some lessons from this new attempt at very early cosmology, whether it succeeds or it fails. As I can see, the main lessons to be drawn are the following:

- Our Universe did not have to emerge, together with space and time, from a singularity; in string theory, the singularity should be fake, either because it is tamed by finite-string-size effects, or because it simply signals the need for new degrees of freedom in the description of physics at very short distances;

- Because string theory is an extension of GR, inflation is possible in that context even in the absence of potential energy (i.e. of an effective cosmological constant); actually, inflation is very natural and easy to achieve, being a consequence of the duality symmetries of the string-cosmology equations;

- Inflation in string cosmology can be related, mathematically, to the problem of gravitational collapse in GR; as such, it is a generic phenomenon, once the assumption of asymptotic past triviality is made; furthermore, the curvature scale and the coupling at the onset of PBB inflation are arbitrary classical moduli;

- The Universe did not have to start hot! A hot Universe can emerge from a cold one thanks to quantum particle production in inflationary backgrounds;

- PBB cosmology predicts a rich spectrum of perturbations with different spectra depending on each perturbation's "pump" field and on its evolution in the PBB era; observable relics of these perturbations may serve as a window on physics in the pre-bangian Universe all the way down to the string/Planck scale;

- The simplest PBB models either predict too small perturbations at large scales, or a spectrum of isocurvature perturbations which may be already "experimentally challenged" (as Rocky Kolb would kindly say);

- The exit problem still remains the hardest theoretical challenge to the whole idea of PBB cosmology;

- Hopefully, the combination of the above-mentioned experimental and theoretical challenges will be able to tell us whether the PBB idea is just doomed, or whether parts of it should be kept while searching for a better scenario; it should also suggest new avenues for physics-driven research in string/M-theory; 
- Last but not least, the PBB idea has taught us that we need not lock ourselves into preconceived ideas in cosmology (cf. "the big bang is the beginning of time", "inflation needs a scalar potential"); rather, we should contemplate as wide a range of theoretically sound possibilities as we can in order for Nature to choose, at best, one of them.

\section{ACKNOWLEDGEMENTS}

I am very grateful to Pierre Binétruy and Richard Schaeffer for having invited me to such a pleasant and stimulating school, to François David for the perfect organization, and to all the students for their patience in listening (after 5 weeks of courses!) and for their interesting questions. 


\section{References}

[1] Lidsey J. E., Wands David and Copeland E. J., hep-th/9909061, to appear in Phys. Rep.

[2] Gasperini M. and Veneziano G., Phys. Rep., in preparation.

[3] http://www.to.infn.it/ gasperin/.

[4] Polchinski J., "String Theory", Cambridge University Press, 1998.

[5] Greene B., "The Elegant Universe", Norton W. W. and Co., New York \& London, 1999.

[6] Veneziano G., "A new approach to semiclassical gravitational scattering", in Proc. 2nd Paris Cosmology Colloquium, Paris, June 1994, eds. de Vega H.J. and Sanchez N., WSPC, Singapore, 1995, p. 322.

[7] Kolb E. W. and Turner M. S., "The Early Universe", Addison-Wesley, Redwood City, CA, 1990

Linde A. D., "Particle Physics and Inflationary Cosmology", Harwood, New York, 1990.

[8] Brustein R. and Steinhardt P. J., Phys. Lett. B302 (1993) 196.

[9] Veneziano G., Europhys. Lett. 2 (1986) 133; "The Challenging Questions", in Proc. Erice, 1989, ed. Zichichi A., Plenum Press, New York, 1990, p. 199.

[10] See, e.g., Witten E., Nucl. Phys. B443 (1995) 85;

Horava P. and Witten E., Nucl. Phys. B460 (1996) 506.

[11] Witten E., Phys. Lett. B149 (1984) 351.

[12] Taylor T. R. and Veneziano G., Phys. Lett. B213 (1988) 459.

[13] Damour T. and Polyakov A. M., Nucl. Phys. B423 (1994) 532; Gen. Rel. Grav. 26 (1994) 1171.

[14] Veneziano G., Phys. Lett. B265 (1991) 287.

[15] Gasperini M. and Veneziano G., Astropart. Phys. 1 (1993) 317.

[16] Gasperini M. and Veneziano G., Mod. Phys. Lett. A8 (1993) 3701.

[17] Gasperini M. and Veneziano G., Phys. Rev. D50 (1994) 2519.

[18] Tseytlin A. A., Mod. Phys. Lett. A6 (1991) 1721;

Tseytlin A. A. and Vafa C., Nucl. Phys. B372 (1992) 443.

[19] Meissner K.A. and Veneziano G., Phys. Lett. B267 (1991) 33, Mod. Phys. Lett. A6 (1991) 3397

Sen A., Phys. Lett. B271 (1991) 295;

Hassan S. F. and Sen A., Nucl. Phys. B375 (1992) 103;

Gasperini M. and Veneziano G., Phys. Lett. B277 (1992) 256. 
[20] Zeldovich Ya. B. and Novikov I. D., "Structure and Evolution of the Universe", University of Chicago Press, 1982.

[21] Veneziano G., Phys. Lett. B406 (1997) 297.

[22] Buonanno A., Meissner K.A., Ungarelli C. and Veneziano G., Phys. Rev. D57 (1998) 2543;

Feinstein A., Lazkoz R. and Vazquez-Mozo M. A., Phys. Rev. D56 (1997) 5166;

Saygili K., Int. J. Mod. Phys. A14 (1999) 225;

Barrow J. D. and Kunze K. E., Phys. Rev. D56 (1997) 741; ibid. D57 (1998) 2255;

Clancy D., Feinstein A., Lidsey J.E. and Tavakol R., Phys. Lett. B451 (1999) 303;

Kunze K. E., gr-qc/9906073.

[23] Belinskii V. A. and Khalatnikov I. M., Sov. Phys. (JETP) 36 (1973) 591;

Deruelle N. and Langlois D., Phys. Rev. D52 (1995) 2007;

Parry J., Salopek D. S. and Stewart J. M., Phys. Rev. D49 (1994) 2872.

[24] Belinskii V. A., Lifshitz E. M. and Khalatnikov L. M., Sov. Phys. Usp. 13 (1971) 745;

Misner C. W., Phys. Rev. Lett. 22 (1969) 1071.

[25] Barrow J. D. and Dabrowski M. P., Phys. Rev. D57 (1998) 7204.

[26] Penrose R., "Structure of space-time", in Battelle Rencontres, eds. Dewitt C. and Wheeler J.A., Benjamin, New York, 1968.

[27] Bondi H., van der Burg M. G. J. and Metzner A. W. K., Proc. Roy. Soc. Lond. A269 (1962) 21;

Sachs R. K., Proc. Roy. Soc. A 270 (1962) 103;

Penrose R., Proc. Roy. Soc. Lond. A284 (1965) 159.

[28] See, e.g., Landau L. and Lifshitz E., "The Classical Theory of Fields", Pergamon Press, Oxford, 1962.

[29] Penrose R., Phys. Rev. Lett. 14 (1965) 57;

Hawking S. W. and Penrose R. R., Proc. Roy. Soc A314 (1970) 529.

[30] Christodoulou D., Commun. Math. Phys. 105 (1986) 337.

[31] Buonanno A., Damour T. and Veneziano G., Nucl. Phys. B543 (1999) 275.

[32] Christodoulou D., Comm. Math. Phys. 109 (1987) 613.

[33] Turner M. and Weinberg E., Phys. Rev. D56 (1997) 4604;

Kaloper N., Linde A. and Bousso R., Phys. Rev. D59 (1999) 043508.

[34] Linde A., Phys. Lett. 129B (1983) 177.

[35] Ghosh A., Pollifrone G. and Veneziano G., Phys. Lett. B440 (1998) 20.

[36] Choptuik M. W., Phys. Rev. Lett. 70 (1993) 9. 
[37] Christodoulou D., Commun. Pure Appl. Math. 44 (1991) 339.

[38] Christodoulou D., Commun. Pure Appl. Math. 46 (1993) 1131.

[39] Maharana J., Onofri E. and Veneziano G., JHEP 04 (1998) 004;

Chiba T., Phys. Rev. D59 (1999) 083508.

[40] Ungarelli C., private communication.

[41] See, e.g., Mukhanov V. F., Feldman A. H. and Brandenberger R. H., Phys. Rep. 215 (1992) 203.

[42] Brustein R., Gasperini M. and Veneziano G., Phys. Lett. B431 (1998) 277.

[43] Grishchuk L. P. and Sidorov Y. V., Class. Quant. Grav. 6 (1989) L161;

Grishchuk L. P., in Proc. Workshop on Squeezed States and Uncertainty Relations, College Park, Maryland, eds. Han D., Kim Y. K. and Zachary W. W., NASA Conf. Pub. No. 3135, 1992, p. 329.

[44] Veneziano G., in String Gravity and Physics at the Planck Energy Scale, Erice, 1995, eds. Sanchez N. and Zichichi A., Kluwer Academic Publishers, Boston, 1996, p. 285;

Gasperini M. , ibid., p. 305.

[45] Brustein R., Gasperini M., Giovannini M. and Veneziano G., Phys. Lett. B361 (1995) 45;

Brustein R. et al., Phys. Rev. D51 (1995) 6744.

[46] Astone P. et al., Phys. Lett. B385 (1996) 421;

Allen B. and Brustein B., Phys. Rev. D55 (1997) 970;

Allen B. and Romano J.D., Phys. Rev. D59 (1999) 102001;

Ungarelli C. and Vecchio A., gr-qc/9911104.

[47] Grishchuk L. P., gr-qc/9903079.

[48] Maggiore M., gr-qc/9909001, to appear in Phys. Rep.

[49] Brustein R. et al., Phys. Rev. D51 (1995) 6744.

[50] Gasperini M., gr-qc/9910019.

[51] Ratra B., Astrophys. J. Lett. 391 (1992) L1.

[52] Gasperini M., Giovannini M. and Veneziano G., Phys. Rev. Lett. 75 (1995) 3796;

Lemoine D. and Lemoine M., Phys. Rev. D52 (1995) 1955.

[53] Kulsrud R. M., Cen R., Ostriker J. P. and Ryu D., Ap. J. 480 (1997) 481.

[54] Copeland E.J., Easther R. and Wands D., Phys. Rev. D56 (1997) 874;

Copeland E.J., Lidsey J.E. and Wands D., Nucl. Phys. B506 (1997) 407.

[55] Buonanno A., Meissner K. A., Ungarelli C. and Veneziano G., JHEP 1 (1998) 4. 
[56] Durrer R., Gasperini M., Sakellariadou M. and Veneziano G., Phys. Lett. B436 (1998) 66, Phys. Rev. D59 (1999) 043511.

[57] Smoot G. F. et al., Ap. J. 396 (1992) L1;

Bennet C. L. et al., Ap. J. 430 (1994) 423.

[58] Melchiorri A., Vernizzi F., Durrer R. and Veneziano G., Phys. Rev. Lett. 83 (1999) 4464.

[59] Mauskopf P. D., et al., astro-ph/9911444.

[60] Brustein R. and Hadad M., Phys. Rev. D57 (1998) 725.

[61] Gasperini M. and Giovannini M., Phys. Lett. B301 (1993) 334, Class. Quant. Grav. 10 (1993) L133;

Brandenberger R., Mukhanov V. and Prokopec T., Phys. Rev. Lett. 69 (1992) 3606, Phys. Rev. D48 (1993) 2443.

[62] Brustein R. and Veneziano G., Phys. Lett. B329 (1994) 429;

Kaloper N., Madden R. and Olive K.A., Nucl. Phys. B452 (1995) 677, Phys. Lett. B371 (1996) 34;

Easther R., Maeda K. and Wands D., Phys. Rev. D53 (1996) 4247.

[63] Giovannini M., Phys. Rev. D57 (1998) 7223.

[64] Brustein R. and Madden R., Phys. Lett. B410 (1997) 110, Phys. Rev. D57 (1998) 712, JHEP 9907 (1999) 006.

[65] Meissner K.A. and Veneziano G., Mod. Phys. Lett. A6 (1991) 3397.

[66] Gasperini M., Maharana J. and Veneziano G. , Phys. Lett. B296 (1992) 51.

[67] See, e.g., Douglas M. R., hep-th/9901146, and references therein.

[68] Gasperini M., Maharana J. and Veneziano G., Nucl. Phys. B472 (1996) 349;

Gasperini M. and Veneziano G., Gen. Rel. Grav. 28 (1996) 1301;

Kehagias A.A. and Lukas A., Nucl. Phys. B477 (1996) 549;

Buonanno A. et al., Class. Quant. Grav. 14 (1997) L97.

[69] Gasperini M., Maggiore M. and Veneziano G., Nucl. Phys. B494 (1997) 315.

[70] Kiritsis E. and Kounnas C., gr-qc/9509017, "String Gravity and Cosmology: Some New Ideas", in Proc. of the Four Seas Conference, Trieste, 1995.

[71] Maggiore M. and Riotto A., Nucl. Phys. B548 (1999) 427.

[72] Ghosh A., Madden R. and Veneziano G., hep-th/9908024.

[73] Antoniadis I., Rizos J. and Tamvakis K., Nucl. Phys. B415 (1994) 497.

[74] Easther R. and Maeda K., Phys. Rev. D54 (1996) 7252. 
[75] Brustein R. and Madden R., Phys. Rev. D57 (1998) 712;

Brandenberger R., Easther R. and Maia J., JHEP 9808 (1998) 007;

Foffa S., Maggiore M. and Sturani R., Nucl. Phys. B552 (1999) 395;

Cartier C., Copeland E. J. and Madden R., hep-th/9910169.

[76] Madden R., private communication.

[77] Bekenstein J. D., Phys. Rev. D23 (1981) 287 and D49 (1994) 1912, and references therein.

[78] 't Hooft G. , gr-qc/9321026, in Abdus Salam Festschrift: A Collection of Talks, eds. Ali A., Ellis J. and Randjbar-Daemi S., World Scientific, Singapore, 1993;

Susskind L., J. Math. Phys. 36 (1995) 6377, and references therein.

[79] Bekenstein J. D., Int. J. Theor. Phys. 28 (1989) 967.

[80] Fischler W. and Susskind L., hep-th/9806039.

[81] Bousso R., JHEP 07 (1999) 004 and 06 (1999) 028.

See also Flanagan E.E., Marolf D. and Wald R.M., hep-th/9908070.

[82] Easther R. and Lowe D.A., Phys. Rev. Lett. 82 (1999) 4967.

[83] Veneziano G., Phys. Lett. B454 (1999) 22 and hep-th/9907012.

[84] Bak D. and Rey S., hep-th/9902173.

[85] Kaloper N. and Linde A., Phys. Rev. D60 (1999) 103509.

[86] Brustein R. and Veneziano G., hep-th/9912055.

[87] Carr B. J. and Hawking S. W., Mon. Not. Roy. Astr. Soc. 168 (1974) 399;

Carr B. J., Astrophys. J. 201 (1975) 1;

Novikov I. D. and Polnarev A. G., Astron. Zh. 57 (1980) 250 [ Sov. Astron. 24 (1980) 147 .

[88] see, e.g., Penrose R., "The Emperor's new mind”, Oxford University Press, New York, 1989, Chapter 7.

[89] Brustein R., gr-qc/9904061;

see also Brustein R., Foffa S. and Sturani R. R., hep-th/9907032.

[90] Lukas A., Ovrut B.A. and Waldram D., Phys. Lett. B393 (1997) 65, Nucl. Phys. B495 (1997) 365;

Larsen F. and Wilczek F., Phys. Rev. D55 (1997) 4591;

Kaloper N., Phys. Rev. D55 (1997) 3394;

Lu H., Mukherji S. and Pope C.N., Phys. Rev. D55 (1997) 7926;

Poppe R. and Schwager S., Phys. Lett. B393 (1997) 51;

Lukas A. and Ovrut B. A., Phys. Lett. B437 (1998) 291;

Kaloper N., Kogan I. and Olive K. A., Phys. Rev. D57 (1998) 7340; 
Banks T., Fischler W. and Motl L., JHEP 9901 (1999) 019;

Feinstein A. and Vazquez-Mozo M. A., hep-th/9906006. 